\title{
Antileishmanial Activity of BNIPDaoct- and BNIPDanon-loaded Emulsomes on Leishmania infantum Parasites
}

\author{
Zeynep Islek ${ }^{1,2}$, Mehmet Hikmet Ucisik ${ }^{1,3,4 *}$, Elif Keskin ${ }^{5}$, Bilgesu Onur Sucu ${ }^{5,6}$, \\ Ana G. Gomes-Alves ${ }^{7,8,9}$, Ana M. Tomás ${ }^{7,8,10}$, Mustafa Guzel ${ }^{5,11}$ and Fikrettin Sahin ${ }^{1}$
}

${ }^{1}$ Department of Genetics and Bioengineering, Faculty of Engineering, Yeditepe University, Istanbul, Turkey, ${ }^{2}$ Department of Pharmaceutical Technology, Faculty of Pharmacy, Istanbul Health and Technology University, Istanbul, Turkey, ${ }^{3}$ Department of Biomedical Engineering, School of Engineering and Natural Sciences, Istanbul Medipol University, Istanbul, Turkey, ${ }^{4}$ Regenerative and Restorative Medicine Research Center (REMER), Research Institute for Health Sciences and Technologies (SABITA), Istanbul Medipol University, Istanbul, Turkey, ${ }^{5}$ Center of Drug Discovery and Development, Research Institute for Health Sciences and Technologies (SABITA), Istanbul Medipol University, Istanbul, Turkey, ${ }^{6}$ Medical Laboratory Techniques, Vocational School of Health Services, Istanbul Medipol University, Istanbul, Turkey, ${ }^{7}$ ISS-Instituto de Investigação e Inovação Em Saùde, Universidade Do Porto, Porto, Portugal, ${ }^{8}$ IBMC-Instituto de Biologia Molecular e Celular, Universidade Do Porto, Porto, Portugal, ${ }^{9} \mathrm{CEB}$-Centro de Engenharia Biológica, Universidade Do Minho, Braga, Portugal, ${ }^{10}$ ICBAS-Instituto de Ciências Biomèdicas Abel Salazar, Universidade Do Porto, Porto, Portugal, ${ }^{11}$ Department of Medical Pharmacology, International School of Medicine, Istanbul Medipol University, Istanbul, Turkey

\section{OPEN ACCESS}

Edited by:

Felipe Alves de Almeida Juiz de Fora Federal University, Brazil

Reviewed by:

Selvaraj Kunjiappan, Kalasalingam University, India Marcus Scotti,

Federal University of Paraíba, Brazil Patricia Sampaio Tavares Veras, Gonçalo Moniz Institute (IGM), Brazil

${ }^{*}$ Correspondence:

Mehmet Hikmet Ucisik mehmet.ucisik@yeditepe.edu.tr

Specialty section: This article was submitted to Biomedical Nanotechnology, a section of the journal

Frontiers in Nanotechnology

Received: 10 September 2021 Accepted: 13 December 2021

Published: 28 January 2022

Citation:

Islek Z, Ucisik MH, Keskin E, Sucu BO, Gomes-Alves AG, Tomás AM, Guzel M and Sahin F (2022) Antileishmanial Activity of BNIPDaoct- and BNIPDanon-loaded Emulsomes on Leishmania infantum Parasites. Front. Nanotechnol. 3:773741. doi: 10.3389/fnano.2021.773741
Among bisnaphthalimidopropyl (BNIP) derivatives, BNIPDaoct and BNIPDanon recently came forward with antileishmanial activities beyond the standard, commercialized antileishmanial therapies. However, high-level toxicity on macrophages plus poor aqueous solubility and poor bioavailability of the compounds limit their application in therapies. Addressing these limitations, the present study introduces BNIPDaoct- and BNIPDanon-loaded emulsomes as lipid-based nanocarrier systems. Accordingly, emulsome formulations were prepared with the presence of BNIP compounds. The average diameters of BNIPDaoct- and BNIPDanon-loaded emulsomes were found as 363.1 and $337.4 \mathrm{~nm}$, respectively; while empty emulsomes differed with a smaller average particle diameter, i.e., $239.1 \mathrm{~nm}$. All formulations exhibited a negative zeta potential value. The formulations achieved the encapsulation of BNIPDaoct and BNIPDanon at approximately $0.31 \mathrm{mg} / \mathrm{ml}(501 \mu \mathrm{M})$ and $0.24 \mathrm{mg} / \mathrm{ml}(387 \mu \mathrm{M})$, respectively. The delivery of BNIP within the emulsomes improved the antileishmanial activity of the compounds. BNIPDaoct-loaded emulsome with 50\% inhibitory concentration $\left(\mathrm{IC}_{50}\right)$ value of $0.59 \pm 0.08 \mu \mathrm{M}$ was in particular effective against Leishmania infantum promastigotes compared to free BNIPDaoct $(0.84 \pm 0.09 \mu \mathrm{M})$, free BNIPDanon $(1.85 \pm$ $0.01 \mu \mathrm{M})$, and BNIPDanon-loaded emulsome $(1.73 \pm 0.02 \mu \mathrm{M})$. Indicated by at least $\geq$ 2 -fold higher $50 \%$ cytotoxic concentration $\left(\mathrm{CC}_{50}\right)$ values, the incorporation of BNIP into
Abbreviations: AmpB, amphotericin B; BNIP, bisnapthalimidopropyl; BNIPDaoct, BNIP diaminooctane; BNIPDanon, BNIP diaminononane; $\mathrm{CC}_{50}$, half maximal cytotoxic concentration; $\mathrm{CL}$, cutaneous leishmaniasis; CLSM, confocal laser scanning microscopy; DBU, 1,8-diazabicyclo[5.4.0] undec-7-ene; DMSO, dimethyl sulfoxide; DIEA, 1,8-naphthalic anhydride, 1,3diaminopropane, $N, N$-diisopropylethylamine; FBS, fetal bovine serum; PBS, phosphate-buffered saline; $\mathrm{IC}_{50}$, half maximal inhibitory concentration; iFBS, inactivated fetal bovine serum; L. infantum, Leishmania infantum; LiSIR2RP1, Leishmania infantum cytosolic SIR2-related protein one; MCL, mucocutaneous leishmaniasis; PDI, polydispersity index; PLA-PEG, poly(lactic acid)-polyethylene glycol; VL, visceral leishmaniasis; PLGA, poly(lactic-co-glycolic acid); RES, reticuloendothelial system; SEM, scanning electron microscope; SI, selectivity index. 
emulsomes significantly reduced the toxicity of BNIPs against macrophages, corresponding to up to 16 -fold improvement in selectivity index $\left(C_{50} / C_{50}\right)$ for $L$. infantum promastigotes. The infection rates of macrophages were determined using dual-fluorescent flow cytometry as $68.6 \%$. Both BNIP formulations at concentration of $1.87 \mu \mathrm{M}$ reduced the parasitic load nearly to $40 \%$, whereas BNIPDaoct-loaded emulosmes could further decrease the parasitic load below $20 \%$ at $7.5 \mu \mathrm{M}$ and above. In conclusion, the incorporation of BNIPDaoct and BNIPDanon into emulsomes results in water-soluble dispersed emulsome formulations that do not only successfully facilitate the delivery of BNIP compounds into the parasites and the Leishmania-infected macrophages in vitro but also enhance antileishmanial efficacy as proven by the decline in $\mathrm{IC}_{50}$ values. The selectivity of the formulation for $L$. infantum parasites further contributes to the challenging safety profile of the compounds. The promising in vitro antileishmanial efficacy of BNIP-loaded emulsomes highlights the potential of the system for the future in vivo studies.

Keywords: bisnapthalimidopropyl (BNIP) derivatives, Leishmania infantum, nanocarrier, emulsome, antileishmanial therapy, infected macrophages, BNIPDaoct, BNIPDanon

\section{HIGHLIGHTS}

Emulsome is considered as a prominent drug delivery strategy to overcome the drug solubility and toxicity limitations of bisnapthalimidopropyl (BNIP) derivatives for their potential use in antileishmanial therapy.

Emulsomes loaded with BNIPDaoct and BNIPDanon with average diameters above $300 \mathrm{~nm}$ achieved concentrations as high as $0.31 \mathrm{mg} / \mathrm{ml}(501 \mu \mathrm{M})$ and $0.24 \mathrm{mg} / \mathrm{ml}(387 \mu \mathrm{M})$, respectively.

Emulsomes enhanced the antileishmanial activity of BNIPDaoct and BNIPDanon on both Leishmania infantum parasites and Leishmania-infected macrophages, thereby reducing their toxicities on macrophages.
BNIPDaoct-loaded emulsome with the $\mathrm{IC}_{50}$ value of $0.59 \pm$ $0.08 \mu \mathrm{M}$ was in particular effective against Leishmania infantum promastigotes compared to BNIPDaoct $(0.84 \pm$ $0.09 \mu \mathrm{M})$, BNIPDanon $(1.85 \pm 0.01 \mu \mathrm{M})$, and BNIPDanonloaded emulsome $(1.73 \pm 0.02 \mu \mathrm{M})$.

Delivered within the emulsomes, the selectivity of the BNIP compounds has been improved up to 16 -fold $\left(\mathrm{CC}_{50} / \mathrm{IC}_{50}\right)$ for Leishmania infantum promastigotes.

\section{INTRODUCTION}

Leishmaniasis is a neglected tropical disease caused by the protozoan parasites of the genus Leishmania and affects

\section{- Delivery of BNIP derivatives to Leishmania-infected Macrophages within Emulsomes -}

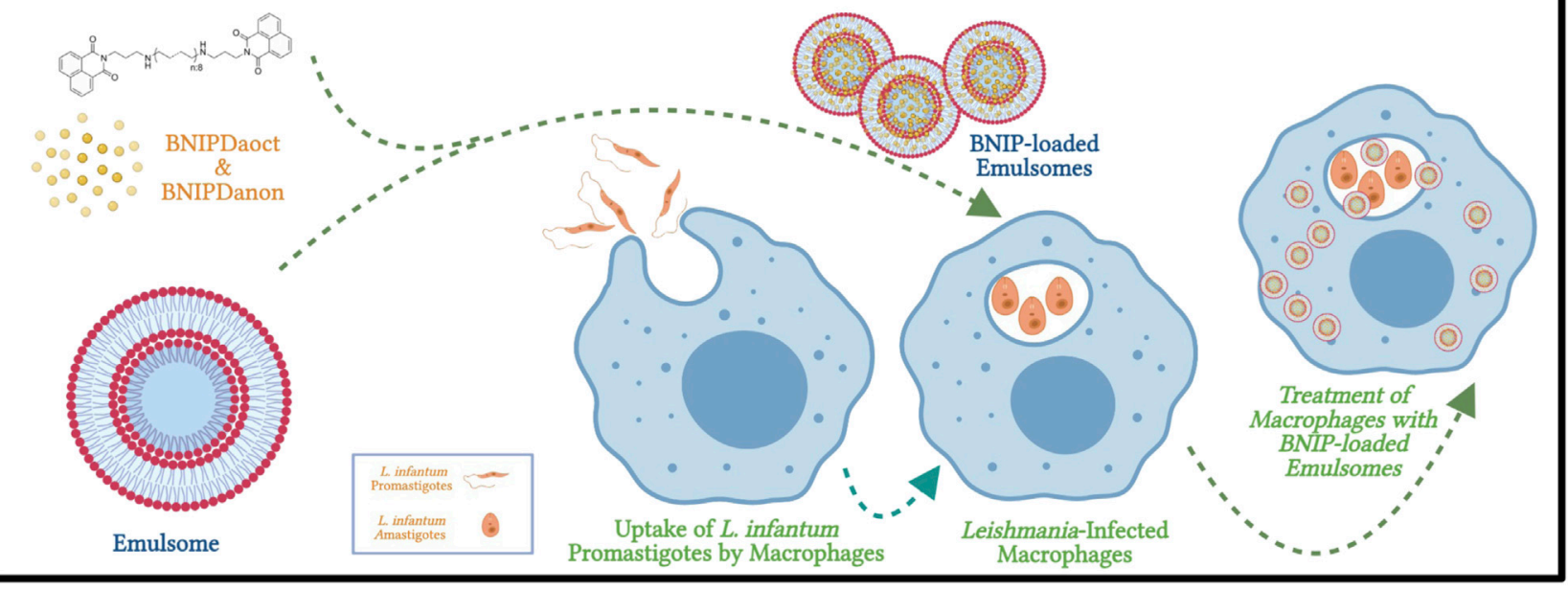

GRAPHICAL ABSTRACT | Graphical abstract illustrates the framework of the study. The figure was created with Biorender.com. 
BNIPDaoct<smiles>O=C1c2cccc3cccc(c23)C(=O)N1CCCNCCCCCCCCNCCCN1C(=O)c2cccc3cccc(c23)C1=O</smiles><smiles>CC(C)C[C@@H](C)Cc1ccccc1</smiles>

FIGURE 1 | Scheme of BNIPDaoct and BNIPDanon.

numerous species including humans, where the infection begins with a bite of phlebotomine sandflies. There are over 20 species of Leishmania, which survive and replicate as either extracellular promastigotes in sandflies or intracellular amastigotes inside mammalian macrophages (Rodrigues et al., 2016). Leishmaniasis is endemic in 98 tropical and subtropical countries with more than 350,000 people at risk as it is estimated that 1.3 million new cases of leishmaniasis occur every year (World Health Organization, 2013). Clinical manifestations of leishmaniasis are divided into three clinical forms: cutaneous leishmaniasis (CL), mucocutaneous leishmaniasis (MCL), and visceral leishmaniasis (VL) known as "Kala Azar", where the latter is fatal when left untreated.

The available treatment options of leishmaniasis rely on pentavalent antimonials-i.e., sodium stibogluconate (pentostan) and meglumine antimoniate (glucantim), pentamidine, conventional amphotericin B (AmpB) deoxycholate, miltefosine, paramomycin (aminosidine), micellar formulation of AmpB (fungizone), AmpB lipid complex (Abelcet), and liposomal AmpB (AmBisome) (Sundar et al., 2002a; Sundar et al., 2002b; Sundar et al., 2003; Köse et al., 2004; Sundar et al., 2004; Murray et al., 2005; Sundar and Chatterjee, 2006; Tiuman et al., 2011; Sundar and Chakravarty, 2013; Aronson, 2017). However, these therapies have certain drawbacks associated with 1) their limited efficacy on parasites (Gupta et al., 2013), 2) multiple adverse effects due to their low therapeutic index (Al-Natour, 2009), 3) requirement for careful and slow intravenous administration due to their toxicities (e.g., AmpB) (Freitas-Junior et al., 2012), and 4) drug resistance developed against the therapy (Sundar et al., 2002a, 2003; Ouellette et al., 2004; Murray et al., 2005; Croft et al., 2006; Sundar and Chatterjee, 2006; Gupta et al., 2007, 2013; Natera et al., 2007; Chakravarty and Sundar, 2010). Moreover, low water solubility and poor bioavailability are the other drawbacks limiting the use of current therapies and further increase the need for new medical therapies.

Therefore, the success in anti-leishmanial therapy largely lies on development of alternative chemotherapy strategies and discovery of new drug molecules with improved characteristics. Several studies reported novel drug candidates with promising efficacy against leishmaniasis (Smith et al., 2000; Kumari et al., 2008; Jain and Jain, 2013; Makala and Baban, 2014).
Among these potential therapeutic agents, bisnapthalimidopropyl (BNIP) derivatives, formulated by two naphthalimide groups linked with a natural polyamine chain, recently came forward with antileishmanial activities that even surpass the standard, commercialized AmpB therapy (Tavares et al., 2012). BNIP derivatives act as $\mathrm{NAD}^{+}$competitive silent regulator inhibitors, known as sirtuins, and display selectivity for Leishmania species by preferentially inhibiting the Leishmania infantum cytosolic SIR2-related protein 1 (LiSIR2RP1), i.e., the sirtuin of L. infantum (Tavares et al., 2010, 2012). Structural difference between the parasitic and human sirtuins is recognized as the reason for up to 17-fold selectivity (Tavares et al., 2010). BNIP derivatives displayed anti-proliferative effects on the life cycle of $L$. infantum and induced the death of promastigote forms by apoptosis (Tavares et al., 2005; Oliveira et al., 2007; Tavares et al., 2010). BNIP derivatives were found more effective than the standard AmpB in reducing the spleen parasite load under a short period of treatment (Tavares et al., 2012).

With two naphthalimide groups linked by a polyamine chain of different sizes and side groups, BNIP derivatives may display varieties in both molecular structure and antileishmanial activity. For instance, BNIP diaminooctane (BNIPDaoct) and BNIP diaminononane (BNIPDanon) with their eight- and ninecarbon chain polyamine chains, respectively (Figure 1), were shown to have $50 \%$ inhibitory concentration $\left(\mathrm{IC}_{50}\right)$ values of $0.78 \pm 0.05 \mu \mathrm{M}$ and $0.78 \pm 0.10 \mu \mathrm{M}$ against $L$. infantum promastigotes, respectively (Oliveira et al., 2007). However, the bioactivity of the compound is not limited with Leishmania promastigotes and amastigotes. High bioactivity of the compounds leads also toxicity on macrophages. On the other hand, hydrophobic characteristics of the compounds result in low aqueous solubility and bioavailability. Furthermore, intracellular localization of the parasite within the macrophages is the other challenge restraining the antileishmanial effect of the therapy on the target site.

Drug delivery systems together with nanoparticle technology is considered as a prominent strategy to overcome drug solubility problems and toxicity of antileishmanial agents such as AmpB (Ambisome $^{\mathrm{TM}}$ and Fungizone ${ }^{\mathrm{TM}}$ ) (Gupta and Vyas, 2007; Adlermoore and Proffitt, 2008; Sundar and Jaya, 2010; Croft and Olliaro, 2011; Costa Lima et al., 2012a; Costa Lima et al., 2012b; Mohamed-Ahmed et al., 2012) and, hence, are also 
thought to be suitable for BNIP compounds. The delivery of BNIP derivatives within drug delivery systems may decrease side effects of the compounds on macrophages and improve the efficacy of the therapy. With this aim, polymeric nanoparticles including poly(lactic-co-glycolic acid) (PLGA) (Costa Lima et al., 2012a) and copolymer poly(lactic acid)-polyethylene glycol (PLA-PEG) (Costa Lima et al., 2012b) have been recently used to produce BNIP-loaded nanoparticular systems with improved characteristics. The incorporation of BNIP derivatives into the polymeric nanoparticles, indeed, increased the efficacy in the delivery of compounds into the macrophages, thereby decreasing the level of the interaction of the compounds with the macrophages' membrane and reducing toxicity thereafter (Costa Lima et al., 2012a; Costa Lima et al., 2012b).

Likewise, our study introduces an alternative lipid-based drug delivery system, so-named emulsomes, for the delivery of BNIPDaoct and BNIPDanon compounds to $L$. infantum promastigotes and macrophages infected therewith. Because of the natural feature of lipids, lipid-based drug delivery systems are known to accumulate in the organs of the reticuloendothelial system (RES), instead of the kidney (Date et al., 2007). As parasites are also located in the organs of RES, use of emulsomes are expected to largely reduce toxicity and to improve the antileishmanial efficacy of the compounds in equal or higher extent than their polymeric alternatives.

Emulsomes are biocompatible lipoidal vesicular systems comprising of a solid fat core surrounded by phospholipid (PL) multi-layers (Ucisik et al., 2013a). Like liposomes, emulsomes are stabilized by PL (multi-)layers as outermost structure. Stabilizing the solid core, PLs eliminate the need for surfactants, thereby providing a high degree of biocompatibility (Ucisik et al., 2015a). The solid- or crystalline-state fat or triglyceride core brings emulsomes two major advantages including 1) high load capacity for lipophilic compounds, where the lipophilic cargo can be present in both the PL layers and the solid fat core, and 2) prolonged release of the cargo from the inner solid matrix (Ucisik et al., 2013a; Ucisik et al., 2013b; Bolat et al., 2020). These two features make emulsomes a better alternative to liposomes for delivery of lipophilic compounds such as BNIPDaoct and BNIPDanon.

As a natural feature shared by lipids, emulsomes are expected to achieve accumulation of the drug in the organs of the RES instead of the kidney, which may largely reduce toxicity of BNIP compounds and improve the antileishmanial efficacy of the treatment, as parasites are also located in the organs of RES. Previous studies have verified the capability of the emulsomes as a lipid-based and surfactant-free drug delivery system for a number of lipophilic compounds on various cancer cells (Paliwal et al., 2009; Ucisik et al., 2013a; Ucisik et al., 2013b; Alhakamy et al., 2020; Bolat et al., 2020; Kammath et al., 2020), neuron cells (ElZaafarany et al., 2018; Yilmaz et al., 2020), parasites (Gupta et al., 2007; Gupta and Vyas, 2007; Gill et al., 2011; Pal et al., 2012), fungi (Kretschmar et al., 2001; Vyas et al., 2009), and viruses (Heiati et al., 1997; Lowell et al., 1997; Heiati et al., 1998; Vyas et al., 2006). These findings in the literature highlight the potential of emulsomes as prominent drug delivery system for poorly water-soluble therapeutic agents such as BNIPDaoct and BNIPDanon.
In this study, BNIPDanon- and BNIPDaoct-loaded emulsome formulations were developed to enhance delivery of the compounds to L. infantum parasites and infected macrophages and, by this means, to improve the efficacy of the therapy, thereby minimizing the hazardous effects on healthy macrophages. The anti-leishmanial efficacy of BNIP-loaded emulsomes was evaluated through analysis on cell viability of $L$. infantum parasites and infected macrophages, whereas the analysis on non-infected macrophages questioned the safety of the designed formulation in the in vitro model.

\section{MATERIALS AND METHODS}

\subsection{Materials}

Glyceryl tripalmitate (tripalmitin, purity $\geq 99 \%$ ), 1,2-dipalmitoyl-racglycero-3-phosphocholine (DPPC, 99\%), and cholesterol ( $\geq 99 \%)$ were purchased from Sigma-Aldrich, Germany. Chloroform ( $\geq 99.8 \%)$ was obtained from Fluka Chemika, Germany. All chemicals were used as received without further purification. 1,8-naphthalic anhydride, 1,3diaminopropane, $N, N$-diisopropylethylamine (DIEA), and 1,8diazabicyclo [5.4.0] undec-7-ene (DBU) were purchased from Sigma-Aldrich (Germany). Solvents such as methanol, ethanol, and toluene were purchased as sure seal dry solvents from Sigma-Aldrich, Germany. Dimethyl sulfoxide (DMSO) was purchased from Fisher BioReagents, United States. Resazurin sodium salt (R7017 - 1G) for Alamar Blue Assay was purchased from Sigma-Aldrich, Germany. PKH67 Green Fluorescent Cell Linker Kits and PKH26 Red Fluorescent Cell Linker Kits were obtained from Sigma-Aldrich, Germany.

\subsection{Synthesis of BNIP Derivatives}

Synthesis of BNIP derivatives were accomplished as described by Keskin and others (Keskin et al., 2019). Accordingly, the synthesis involves two-step reactions. Reactions were conducted under an atmosphere of nitrogen, using pre-dried septa. The tips of cannulae were flame-dried under a stream of dry nitrogen gas prior to use. All oxygen- and moisture-sensitive reactions described herein were performed in glassware that was oven dried $\left(110^{\circ} \mathrm{C}, 12 \mathrm{~h}\right)$ then flame dried $\left(\mathrm{N}_{2}\right.$ stream) immediately prior to use.

In the first step, the substitution reaction between 1,8naphthalic anhydride (198 mg, $1 \mathrm{mmol}$ ) and 1,3diaminopropane $(93 \mathrm{mg}, 1.3 \mathrm{mmol})$ at $75^{\circ} \mathrm{C}$ in ethanol $(30 \mathrm{ml})$ as solvent provided the intermediate compound, naphthalimidopropanamine ([2-(3-aminopropyl)-1H-benzo [de] isoquinoline-1,3(2H)-dione]), with $81 \%$ yield. In the second step, the intermediate compound (508 $\mathrm{mg}, 2 \mathrm{mmol}$ ) and 1,8-dibromoctane $(272 \mathrm{mg}, 1 \mathrm{mmol})$ were let into reaction in the presence of DBU base $(152 \mathrm{mg}, 1 \mathrm{mmol})$ in methanol to produce BNIPDaoct. The two-step BNIPDaoct synthesis was achieved overall with $48 \%$ yield (Keskin et al., 2019).

For the synthesis of BNIPDanon, the intermediate compound (508 mg, $2 \mathrm{mmol}$ ) was let into reaction with DIEA (259 mg, $2 \mathrm{mmol})$, and 1,9-dibromononane $(286 \mathrm{mg}, 1 \mathrm{mmol})$ were added to in toluene/ethanol $(1: 1)(60 \mathrm{ml})$ and then were followed by reflux for $48 \mathrm{~h}$. BNIPDanon synthesis was achieved overall with 50\% yield (Keskin et al., 2019). 
For the analysis of products, analytical thin-layer chromatography (TLC) was performed on glass-backed $250 \sim$ plates by visualizing with anisaldehyde and phosphomolybdic acid. $1 \mathrm{H}$ Spectra were recorded at $500 \mathrm{MHz}$ and $13 \mathrm{C}$ spectra at $125 \mathrm{MHz}$, both on a Bruker AM 400 instrument (Bruker, Karlsruhe, Germany). Mass spectra were recorded on a Shimadzu mass spectrometry (MS) system equipped with electron spray ionization source operated in a positive mode (Kyoto, Japan). Mass spectra were recorded on a Shimadzu MS system. In all synthetic methods, appropriate reference methods were used. LC-MS and NMR data of the synthesis products and intermediate compounds are available in the previously published study of Keskin et al. (2019).

\subsection{Preparation of Emulsome Loaded With Bisnaphthalimidopropyl Derivative Compounds}

BNIPDaoct and BNIPDanon-loaded emulsomes have been separately synthesized applying the procedure described before with slight modifications (Ucisik et al., 2013b; Ucisik et al., 2015b). Briefly, the conventional rotary evaporation procedure was used where lipids (i.e., tripalmitin, dipalmitoylphosphatidylcholine, and cholesterol) together with BNIPDaoct/BNIPDanon were first dissolved in organic solvent (i.e., chloroform). Solvent was completely removed. The obtained dry lipid film was rehydrated with an aqueous solution. Ultrasonication was used to homogenize the emulsomes in terms of size and shape. BNIPDaoct- and BNIPDanon-loaded emulsome preparations were centrifuged at $16,100 \mathrm{~g}$ for $10 \mathrm{~min}$ to spin down unincorporated BNIPDaoct/BNIPDanon. The supernatant, i.e., emulsome suspension, was kept at $4^{\circ} \mathrm{C}$ for further characterization and cell culture studies. Empty emulsomes were prepared applying the same procedure without the addition of BNIPDaoct/BNIPDanon.

\subsection{Characterization of Emulsome Formulations}

\subsubsection{Measurement of Size, Polydispersity, and Zeta Potential}

The size distribution, polydispersity index (PDI), mean particle size, and mean zeta potential of the emulsome formulations were determined at $25^{\circ} \mathrm{C}$ by using a zetasizer (Zetasizer Nano ZS, Malvern Instruments Ltd., UK). Prior to the measurement, the emulsome suspensions were diluted with $1 \mathrm{mM} \mathrm{KCl}$ to suitable concentrations. All results were obtained as the average value of triplicate samplings and measurements for each formulation. The dispersity of the formulations in water was additionally investigated under confocal laser scanning microscopy (CLSM).

\subsubsection{Morphological Analysis}

Scanning electron microscope (SEM) (Zeiss EVO-HD-15) was used to analyze size, shape, and morphology of the prepared emulsome formulations. Before imaging, a short-term fixation procedure was applied, as previously described (Bolat et al., 2020). Briefly, emulsome formulations were placed into an aluminum holder and incubated overnight at $4^{\circ} \mathrm{C}$. For the fixation, dried formulations were treated with phosphate-buffered saline (PBS) containing $2.5 \%$ glutaraldehyde for $15 \mathrm{~min}$. Samples washed with distilled water three times in total $10 \mathrm{~min}$. Following gold sputtering (EM ACE200, Leica), the samples were observed under SEM (Zeiss EVO-HD-15).

\subsubsection{Quantification of BNIPDaoct and BNIPDanon Encapsulated in Emulsome Formulations}

Both BNIPDaoct and BNIPDanon content within the emulsome formulations were quantified using the intrinsic fluorescent properties of the compounds, as described by Lima and the colleagues (Costa Lima et al., 2012b). Following excitation at $330 \mathrm{~nm}$, the emitted fluorescence was read at $495 \mathrm{~nm}$ using UVvis spectrophotometer (Spectramax i3 Multi-Mode Microplate Reader Detection Platform). BNIPDaoct and BNIPDanon stock solutions $(1 \mathrm{mg} / \mathrm{ml})$ were prepared in DMSO. Standard samples $(0,5,10,20,50$, and $100 \mu \mathrm{g} / \mathrm{ml})$ were prepared from the stock solutions with successive dilutions in 96-well microplates (NEST Scientific, catalog no. 701001). Both BNIPDanon and BNIPDaoct emulsome formulations were diluted in 1:10 ratios with DMSO. A standard curve was prepared from the emitted fluorescence values of standards. BNIPDanon and BNIPDaoct concentrations in emulsomes were estimated by the read-out of the fluorescence intensity and its corresponding concentration value on the standard curve.

\subsubsection{Encapsulation Efficiency}

Encapsulation efficiency of BNIPDaoct and BNIPDanon emulsome formulations were calculated according to the following equation:

$$
\text { Encapsulation Efficiency }(\%)=\frac{W_{\text {incorporated compound }}}{W_{\text {total }}} \times 100 \%
$$

$W_{\text {incorporated compound }}$ is the amount of BNIPDaoct/ BNIPDanon in the emulsomes; $\boldsymbol{W}_{\text {total }}$ is the amount of BNIPDaoct/BNIPDanon used in the formulation.

\subsection{Parasite and Macrophage Cell Culture} 2.5.1 Parasite Culture Leishmania infantum promastigotes (MHOM/MA/67/ITMAP263) were kindly provided by Dr. Ana M. Tomás [Institute for Molecular and Cell Biology (IBMC), University of Porto (Portugal)]. Leishmania infantum promastigotes were cultured at $25^{\circ} \mathrm{C}$ in RPMI 1640 GlutaMAX (Gibco) supplemented with $10 \%(\mathrm{v} / \mathrm{v})$ inactivated fetal bovine serum (iFBS), penicillin (50 U/ $\mathrm{ml})$, streptomycin $(50 \mu \mathrm{g} / \mathrm{ml})$, and $20 \mathrm{mM}$ HEPES sodium salt ( $\mathrm{pH}$ 7.4) (Sigma-Aldrich, Germany). Infective metacyclic promastigotes were obtained from stationary phase cultures of L. infantum for no longer than seven passages to maintain infectivity. Promastigotes were collected from the pellet by centrifugation at 3,000 $\mathrm{g}$ for $10 \mathrm{~min}$ (Gomes-Alves et al., 2018).

\subsubsection{Macrophage Cell Culture}

The murine macrophage cell line J774A.1 (TIB-67) was purchased from American Type Culture Collection (Rockville, MD). Cells were cultured as a monolayer at $37^{\circ} \mathrm{C}$ in a humidified 
atmosphere containing $5 \% \mathrm{CO}_{2}$, in Dulbecco's modified Eagle's medium supplemented with $10 \%$ fetal bovine serum (FBS) (Invitrogen), $2 \mathrm{mM}$ L-glutamine, penicillin (100 U/ml), and streptomycin $(100 \mu \mathrm{g} / \mathrm{ml})$ (Biological Industries, Beit Haemek, Israel). Cells were maintained in culture by sub-passaging every 3 days.

\subsubsection{Cytotoxicity Assay on Leishmania infantum Promastigotes}

Effects of BNIPDaoct, BNIPDanon, BNIPDaoct-loaded, and BNIPDanon-loaded emulsome formulations on viability of $L$. infantum promastigotes were tested using the standard resazurin assay. Accordingly, L. infantum promastigotes at exponential phase of growth were placed in 96-well plates at $3 \times 10^{5}$ cells per well in completed RPMI medium. L. infantum parasites were exposed to free forms and emulsome formulations of BNIPDaoct/ BNIPDanon at different concentrations $(0-10 \mu \mathrm{M})$ and further incubated for $72 \mathrm{~h}$. Empty emulsome was used as control group to evaluate if emulsome alone result in any alteration on viability of L. infantum promastigotes. At the end of the treatment, $10 \%(\mathrm{v} / \mathrm{v})$ of a $2.5 \mathrm{mM}$ resazurin solution (Sigma)-Alamar Blue was added to each well and fluorescence measured (excitation at $560 \mathrm{~nm}$; emission at $590 \mathrm{~nm}$ ) using a UV-vis spectrophotometer (Spectramax i3 Multi-Mode Microplate Reader Detection Platform). Parasite viabilities were estimated as the percentages compared to the negative control group, where no treatment was applied. Data were analyzed with GraphPad Prism 6.1 software, and the $\mathrm{IC}_{50}$ values were determined (Oliveira et al., 2016).

\subsubsection{Cell Viability Assay on Macrophages}

J774A.1 murine macrophages were incubated in the presence of BNIPDaoct and BNIPDanon in both free forms and emulsome formulations. Cell viabilities were determined using the standard resazurin assay (Sigma-Aldrich, Germany). Briefly, J774A.1 cells were seeded in 96-well plates with a density of 10,000 cells per well. After $24 \mathrm{~h}$ of incubation, cells were treated with different concentrations of BNIPDaoct and BNIPDanon in free forms and with their emulsome formulations. Following the incubation with BNIPDaoct, BNIPDanon, BNIPDaoct-loaded, and BNIPDanonloaded emulsomes for 24,48 , and $72 \mathrm{~h}$, cells were incubated in $10 \%(\mathrm{v} / \mathrm{v})$ of a $2.5 \mathrm{mM}$ resazurin solution (Sigma) for $2 \mathrm{~h}$. The fluorescence intensity was measured (excitation at $560 \mathrm{~nm}$; emission at $590 \mathrm{~nm}$ ) using a UV-vis spectrophotometer (Spectramax i3 Multi-Mode Microplate Reader Detection Platform). Cell viability was calculated as the percentage compared to negative control culture. Data were analyzed with GraphPad Prism 6.1 software and 50\% cytotoxic concentration $\left(\mathrm{CC}_{50}\right)$ values determined.

\subsubsection{Staining of Macrophages and Parasites With Fluorescence Dyes}

J774A.1 macrophages and $L$. infantum promastigotes were stained with PKH26 (Red Fluorescent Cell Linker Kit, SigmaAldrich) and PKH67 (Green Fluorescent Cell Linker Kit, SigmaAldrich), respectively, as described by Islek et al. (2021). Briefly, a total of $10^{7}$ cells $/ \mathrm{ml}$ were used as cell/parasite concentration. Prior to the infection, the promastigotes were examined by flow cytometry (BD FACSCalibur, United States) with a total of 100,000 events to ensure that all parasites are successfully stained with PKH67 dye and can be detected by flow cytometry.

\subsubsection{Flow Cytometry for Cell Viability Analysis of Macrophages Infected With Leishmania infantum Promastigotes}

PKH26-labeled J774A.1 cells were seeded on 12-well plates with a density of 180,000 cells per well and incubated at $37^{\circ} \mathrm{C}$ and $5 \% \mathrm{CO}_{2}$. After $24 \mathrm{~h}$ of incubation, J774A.1 cells were infected with PKH67labeled $L$. infantum promastigotes at a ratio of 1:10. Following $3 \mathrm{~h}$ of co-incubation, non-internalized parasites were removed by gently washing with PBS. After $24 \mathrm{~h}$ of incubation, infected cells were treated with different concentrations of BNIPDaoct, BNIPDanon, and their emulsome formulations for 24 and $48 \mathrm{~h}$. Infection ratios of macrophages were detected by flow cytometry (BD FACSCalibur, United States) according to their relative fluorescence intensities, as previously described in Islek et al. (2021). Green fluorescence (FL1) filter was used for detection of PKH67-labeled parasites (excitation at $490 \mathrm{~nm}$; emission at $502 \mathrm{~nm}$ ), and red fluorescence (FL2) filter was used for PKH26-labeled macrophages (excitation at $551 \mathrm{~nm}$; emission at $567 \mathrm{~nm}$ ). Gates were set for each experiment to optimize the separation of infected cells. To increase the reliability of the statistical data, at least 100,000 cells were collected and read on flow cytometry. The data were confirmed by using microscopical detection and counting method.

\subsection{Statistical Analysis}

GraphPad Prism software (version 6.01) was used to perform statistical analysis. Error bars represent standard error of the mean. The data sets were compared using ordinary one-way ANOVA and two-way ANOVA followed by Tukey's multiple-comparison test. Differences were considered statistically significant at $\left(^{*}\right) p \leq 0.05$, $\left.{ }^{* *}\right) p \leq 0.01,\left({ }^{* *}\right) p \leq 0.001$, and $\left(^{* * *}\right) p \leq 0.0001$.

\section{RESULTS}

\subsection{Characterization of Emulsome Formulations}

\subsubsection{Size, Polydispersity and Zeta Potential}

At least five separate BNIPDaoct- and BNIPDanon-loaded emulsome formulations were analyzed for their size, PDI, and zeta potential characteristics. The average diameters of BNIPDaoct- and BNIPDanon-loaded emulsomes (Table 1) were found as $363.1 \pm 51.7 \mathrm{~nm}$ (PDI: 0.375; conductivity: $0.029 \pm$ $0.003 \mathrm{mS} / \mathrm{cm}$ ) and $337.4 \pm 50.8 \mathrm{~nm}$ (PDI: 0.350 ; conductivity: $0.021 \pm 0.002 \mathrm{mS} / \mathrm{cm})$, respectively; whereas empty emulsomes have a smaller average particle diameter: $239.12 \pm 51.52 \mathrm{~nm}$ (PDI: 0.19; conductivity: $0.027 \pm 0.002 \mathrm{mS} / \mathrm{cm}$ ).

All formulations exhibited a negative zeta potential value (Table 1): empty emulsomes $(-16.74 \pm 4.56 \mathrm{mV})$, BNIPDaoctloaded emulsomes $(-4.77 \pm 6.68 \mathrm{mV})$, and BNIPDanon-loaded emulsomes $(-10.10 \pm 9.76 \mathrm{mV})$. Although BNIPDaoct-loaded emulsomes were distinguished with a less negative zeta potential value close to neutral, no significant difference $(p \geq 0.05)$ was found among the average zeta potential values of empty and BNIP-loaded 
TABLE 1 | The average particle size, polydispersity index, and average zeta potential values for BNIPDaoct- and BNIPDanon-loaded emulsomes.

Formulations
Average

particle size $(\mathrm{nm})$

$239.1 \pm 51.5$
$363.1 \pm 51.7$
$337.4 \pm 50.8$

Polydispersity index

$$
\begin{aligned}
& 0.19 \pm 0.02 \\
& 0.38 \pm 0.07 \\
& 0.35 \pm 0.04
\end{aligned}
$$

Average

zeta potential (mV)

$-16.74 \pm 4.56$

$-4.77 \pm 6.68$

$-10.10 \pm 9.76$

BNIPDanon-loaded emulsome
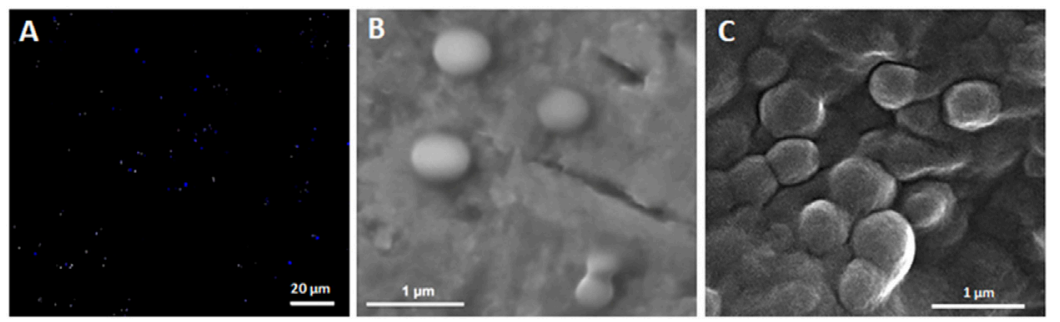

FIGURE 2 |Dispersity, shape, and morphology analysis of emulsomes. (A) CLSM image of BNIPDaoct-loaded emulsomes. Scale bar corresponds to 20 m. (B,C) SEM micrographs of BNIPDaoct- and BNIPDanon-loaded emulsomes, respectively. Scale bars correspond to $1 \mu \mathrm{m}$.
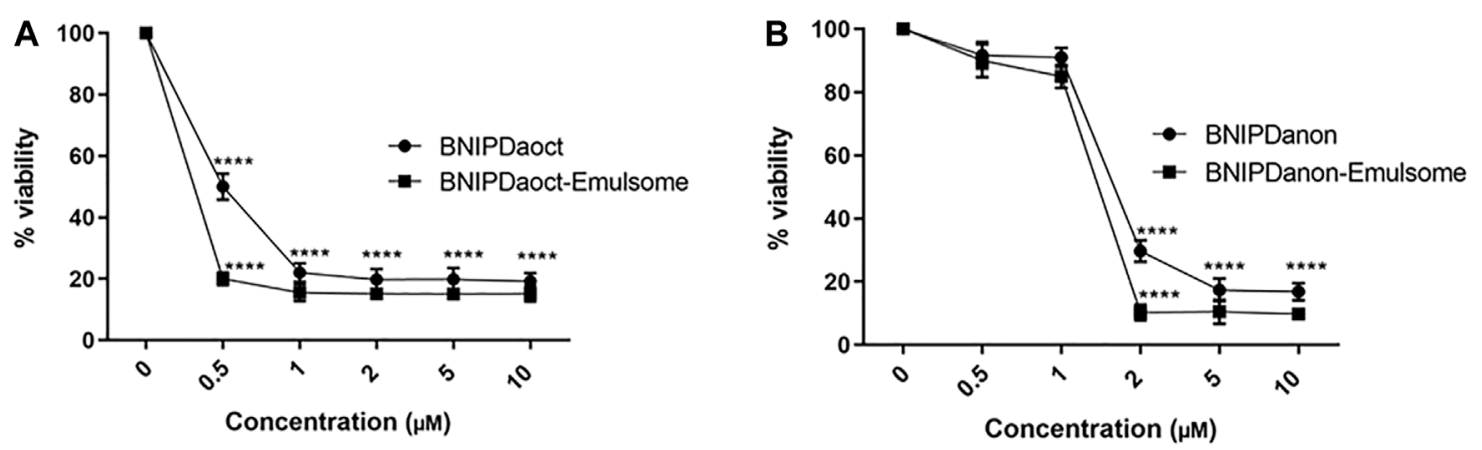

FIGURE 3 | Effect of free BNIP derivatives and BNIP-loaded emulsomes on proliferation of $L$. infantum promastigotes. Viability of Leishmania infantum parasites after $72 \mathrm{~h}$ of treatment with (A) BNIPDaoct and BNIPDaoct-loaded emulsome and (B) BNIPDanon and BNIPDanon-loaded emulsomes. Data represents mean ( $\mathrm{n}=3$ ) \pm SD. Statistical significance was determined using two-way ANOVA with Tukey's multiple-comparison test $\left({ }^{\star} p \leq 0.05,{ }^{\star \star} p \leq 0.01,{ }^{\star \star \star} p \leq 0.001\right.$, and $\left.{ }^{\star \star \star \star} p \leq 0.0001\right)$.

emulsomes. This finding indicates that the incorporation of BNIP derivatives into emulsomes statistically did not cause a significant change in the zeta potential of emulsomes.

\subsubsection{Dispersity, Shape and Morphology}

Incorporated into emulsomes, BNIPDaoct and BNIPDanon with their intrinsic autofluorescence properties emitted fluorescence signal from the solid tripalmitin core of the formulation. The samples displayed dispersed characteristics in aqueous environment as examined by CLSM (Figure 2A). Confirming the structural uniformity, no by-products such as liposomes or lipid aggregates were detected.

The SEM analysis revealed the spherical shape and smooth surface morphology of BNIP-loaded emulsome formulations. In parallel to the DLS analysis, the SEM analysis evidenced that the sizes of BNIP-loaded emulsomes largely vary between 100 and $500 \mathrm{~nm}$ (Figures 2B,C).

\subsubsection{Quantification of BNIPDaoct and BNIPDanon Encapsulated in Emulsomes}

BNIPDaoct and BNIPDanon concentrations in distinct emulsome formulations were estimated in average as $0.31 \mathrm{mg} /$ $\mathrm{ml}(501 \mu \mathrm{M})$ and $0.24 \mathrm{mg} / \mathrm{ml}(387 \mu \mathrm{M})$, respectively.

\subsubsection{Encapsulation Efficiency}

The incorporation of BNIP derivatives into the emulsomes was achieved through the addition of the compounds to the lipid composition during the start of the emulsome preparations. To achieve highest amount of incorporation, both BNIP derivatives were added in excess amounts-ranging from 1:9 to 1:22 in 
TABLE 2 | The half-maximal inhibitory concentration $\left(\mathrm{IC}_{50}\right)$ of BNIPDaoct-loaded emulsome and BNIPDanon-loaded emulsome formulations as compared to the value of free forms of BNIPDaoct and BNIPDanon $\left(\mathrm{IC}_{50}\right.$ values for $72 \mathrm{~h}$ of treatment of Leishmania infantum promastigotes).

\begin{tabular}{lc} 
& $\mathbf{I C}_{\mathbf{5 0}}(\boldsymbol{\mu} \mathbf{M})^{\mathbf{a}}$ \\
\hline BNIPDaoct & $0.84 \pm 0.09$ \\
BNIPDaoct-loaded emulsome & $0.59 \pm 0.08^{\star \star}$ \\
BNIPDanon & $1.85 \pm 0.01$ \\
BNIPDanon-loaded emulsome & $1.73 \pm 0.02^{\text {ns }}$
\end{tabular}

aViability determined by Alamar Blue assay. The results were obtained after treatment of the promastigote form of the parasite with free forms of BNIPDaoct and BNIPDanon, and their emulsome formulations at concentrations $(0.5-10 \mu \mathrm{M})$ for $72 \mathrm{~h}$. Data represents mean $(\mathrm{n}=3) \pm S D$. Statistical significance was determined using ordinary one-way ANOVA, with Tukey's multiple-comparison test (ns: not significant; ${ }^{*} \mathrm{p} \leq 0.05$, ${ }^{* *} \mathrm{p} \leq 0.01$, ${ }^{* * *} \mathrm{p} \leq 0.001$, and ${ }^{* * * *} \mathrm{p} \leq 0.0001$ ).

weight ratio (1:7-1:18 in molar ratio) - relative to total lipid (TL) composition. After the removal of the unincorporated BNIP from the system via centrifugation, encapsulation efficiencies of the final emulsome products were found as $6.2 \%$ and $6.0 \%$ for BNIPDaoct- and BNIPDanon-loaded emulsomes, respectively.

\subsection{Cell Culture Studies}

\subsubsection{Antileishmanial Effect of the BNIP Compounds and BNIP-loaded Emulsomes on Growth of $L$. infantum Promastigotes}

L. infantum promastigotes were treated with free BNIPDaoct, free BNIPDanon, BNIPDaoct-loaded, and BNIPDanon-loaded emulsomes for $72 \mathrm{~h}$ at concentrations ranging from 0.5 to $10 \mu \mathrm{M}$. As shown in Figure 3, all treatment groups inhibited proliferation of parasites in dose-dependent manner. BNIPDaoct concentration of $0.5 \mu \mathrm{M}$ significantly decreased the viability of $L$. infantum promastigotes to exactly 50\% ( $p \leq 0.0001$ ), which became nearly $20 \%$ (i.e., 22\%) when the concentration was increased to $1 \mu \mathrm{M}$ ( $p \leq 0.0001$ ) (Figure 3A). Interestingly, the increase in BNIPDaoct concentrations above $1 \mu \mathrm{M}$ did not cause any significant further change in inhibition of promastigote proliferation (i.e., 19.2\%). On the other hand, $2 \mu \mathrm{M}$ free BNIPDanon concentration significantly decreased the viability of promastigotes sharp to approximately $30 \%$ (i.e., 29.7\%) ( $p \leq$ 0.0001) (Figure 3B). Similarly, at $5 \mu \mathrm{M}$ BNIPDanon concentration, the viability of promastigotes was significantly decreased $(p \leq 0.0001)$ and became close to $15 \%$ (17.4\%). Further increase in BNIPDanon concentration to $10 \mu \mathrm{M}$ did not alter viability of $L$. infantum promastigotes significantly (i.e., 16.8\%).

Releasing the BNIP derivatives gradually into the cytoplasm, emulsome formulations of BNIPDaoct and BNIPDanon induced a gradual decrease in viability of $L$. infantum promastigotes. BNIPDaoct-loaded emulsome with compound concentration equal to $0.5 \mu \mathrm{M}$ significantly decreased the viability of $L$. infantum promastigotes sharp to approximately $20.0 \%$, which was reduced to approximately $15 \%$ (i.e., $15.5 \%$ ) at $1 \mu \mathrm{M}$ ( $p \leq$ 0.0001 ) (Figure 3A). The increase in BNIPDaoct concentrations further from $1 \mu \mathrm{M}$ did not significantly provide further inhibition in proliferation of the promastigote culture with a minimum parasite viability of $15.1 \%$. On the other hand, $2 \mu \mathrm{M}$ BNIPDanonloaded emulsomes concentration was necessary to decrease the viability of promastigotes sharp to approximately $10 \%$ (i.e., 10.3\%) (Figure 3B) $(p \leq 0.0001)$. The increase in concentration of BNIPDanon-loaded emulsomes from 2 to $10 \mu \mathrm{M}$ did not lead to a significant improvement in inhibition of proliferation of promastigotes further $(10.3 \%$ and $9.8 \%$, respectively) (Figure 3B). Empty emulsomes did not show any effect on proliferation of L. infantum promastigotes for $72 \mathrm{~h}$ (data not shown).

$\mathrm{IC}_{50}$ values $(72 \mathrm{~h})$ for BNIPDaoct, BNIPDanon, BNIPDaoctloaded, and BNIPDanon-loaded emulsomes were calculated as $0.84 \pm 0.09 \mu \mathrm{M}, 1.85 \pm 0.01 \mu \mathrm{M}, 0.59 \pm 0.08 \mu \mathrm{M}$, and $1.73 \pm$ $0.02 \mu \mathrm{M}$, respectively (Table 2). The results revealed that, with a lower $\mathrm{IC}_{50}$ value, BNIPDaoct-loaded emulsome is more effective against $L$. infantum promastigotes than the free forms of both BNIPDaoct and BNIPDanon, as well as BNIPDanon-loaded emulsomes. Furthermore, the $\mathrm{IC}_{50}$ value of BNIPDaoct on $L$. infantum promastigotes was found consistent with the value in the literature, i.e., $0.78 \pm 0.05 \mu \mathrm{M}$ (Oliveira et al., 2007). The incorporation of BNIPDaoct into emulsomes seems to increase the antileishmanial efficacy of the compound, as the improvement in the $\mathrm{IC}_{50}$ value of BNIPDaoct upon encapsulation $(0.59 \pm 0.08 \mu \mathrm{M})$ points at a statistical significance $(p \leq 0.01)$ (Table 2). Likewise, a decrease was observed in the $\mathrm{IC}_{50}$ value of BNIPDanon upon encapsulation into emulsomes; however, the alteration from $1.85 \pm 0.01 \mu \mathrm{M}$ to $1.73 \pm 0.02 \mu \mathrm{M}$ was found statistically insignificant (ns).

\subsubsection{Cytotoxicity of Free BNIP and BNIP-loaded Emulsomes on Macrophages}

Following the in vitro evaluation of antileishmanial effect of free BNIP compounds and their emulsome formulations on $L$. infantum promastigotes, cytotoxicity of the compounds and the formulations was separately evaluated on non-infected murine J774A.1 macrophages (Figure 4). Cell viabilities were determined using Alamar Blue Assay for 24, 48, and $72 \mathrm{~h}$.

As shown in Figure 4, when treated with free BNIPDaoct at concentrations $\leq 1.25 \mu \mathrm{M}$, cell viability of non-infected J774A.1 macrophages remained above $95 \%$ for 24,48 , and $72 \mathrm{~h}$. However, when the concentration was increased to $\geq 2.5 \mu \mathrm{M}$, the cell viability was reduced dramatically to $8 \%$ after $24 \mathrm{~h}$ and below $5 \%$ after both 48 and $72 \mathrm{~h}$ compared to the negative control group $(p \leq 0.0001)$ (Figure 4A). The incorporation of BNIPDaoct into emulsomes decreased toxicity of the compound on non-infected macrophages, as the data revealed that BNIPDaoct-loaded emulsomes with concentrations $\leq 15 \mu \mathrm{M}$ did not cause any cytotoxicity on healthy macrophages for 48 and $72 \mathrm{~h}$ (Figure 4B). However, when treated with $30 \mu \mathrm{M}$ BNIPDaoct-loaded emulsomes, the macrophage viability immediately dropped below $6 \%$ after 24,48 , and $72 \mathrm{~h}(p \leq 0.0001)$. At the same concentrations, emulsomes lacking the BNIP did not cause any significant toxicity, as the cell viability remained always higher than $95 \%$ for $72 \mathrm{~h}$.

When treated with free BNIPDanon at concentrations $\leq 2.5 \mu \mathrm{M}$, cell viability of healthy J774A.1 macrophages remained above $87.5 \%$ for 24,48 , and $72 \mathrm{~h}$. However, when the concentration was increased to $\geq 5 \mu \mathrm{M}$, the cell viability was significantly reduced to below $30 \%, 6 \%$, and $15 \%$ after 24 , 48 , and $72 \mathrm{~h}$, respectively ( $p \leq 0.0001$ ) (Figure 4C). A slight 


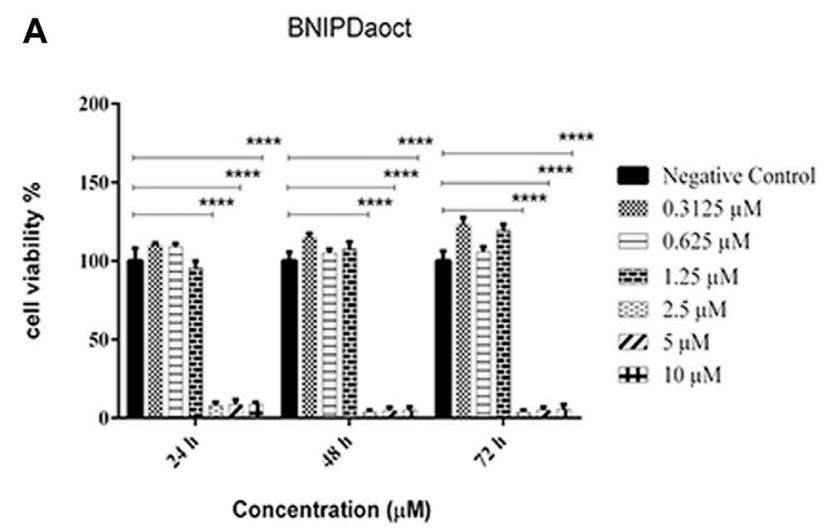

B BNIPDaoct-Emulsome
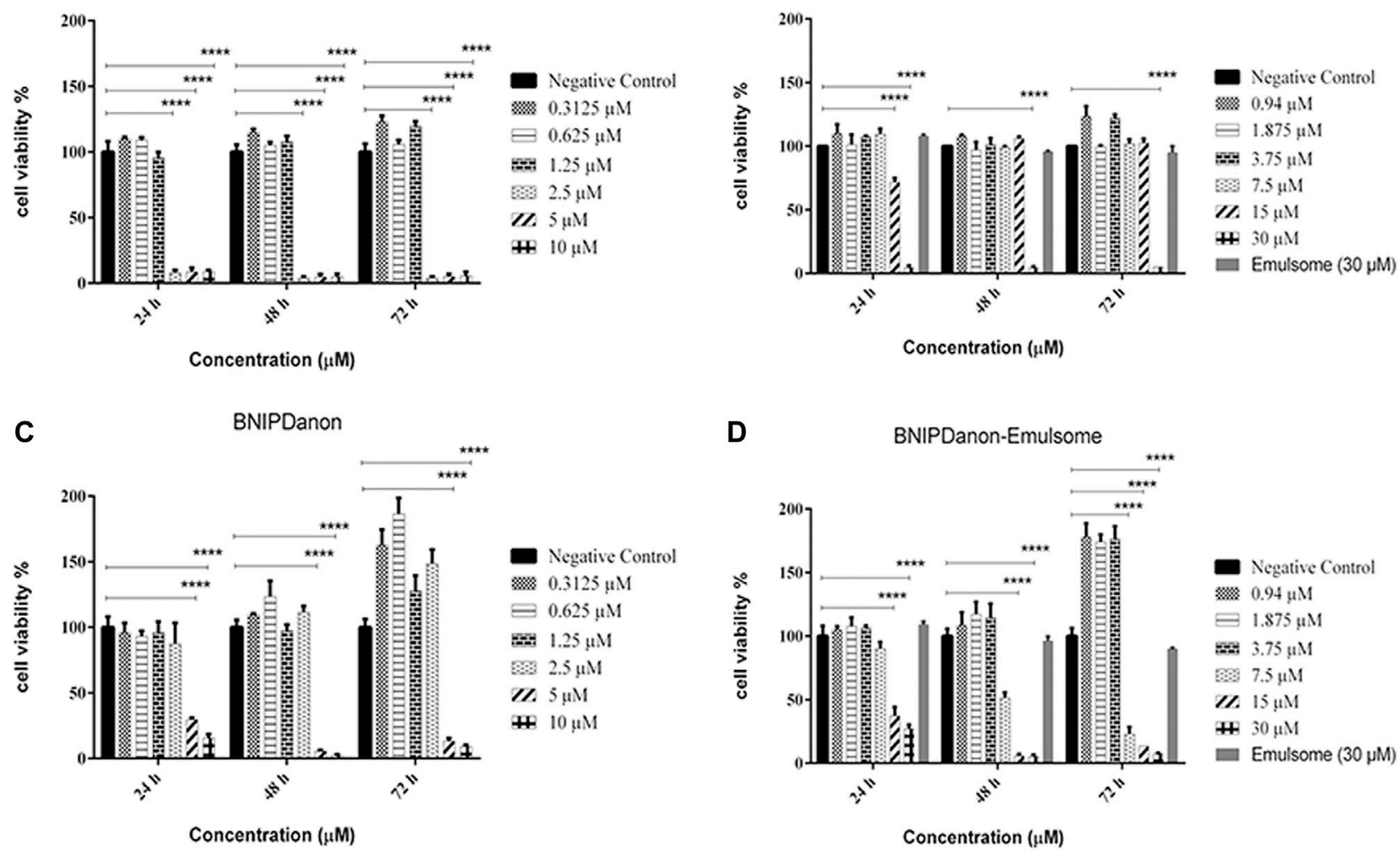

FIGURE 4 | Cytotoxicity of free BNIP and BNIP-loaded emulsomes on macrophages. Cell proliferation analysis of J774A.1 macrophages treated with (A) free BNIPDaoct (in DMSO), (B) BNIPDaoct-loaded emulsomes, (C) free BNIPDanon (in DMSO), and (D) BNIPDanon-loaded emulsomes were investigated at various concentrations $(0.3-30 \mu \mathrm{M})$ for 24,48 , and $72 \mathrm{~h}$ compared to untreated negative control cells. Data represents mean $(n=3) \pm \mathrm{SD}$. Statistical significance was determined using two-way ANOVA with Tukey's multiple-comparison test ( ${ }^{\star} p \leq 0.05,{ }^{\star \star} p \leq 0.01,{ }^{\star \star \star} p \leq 0.001$, and $\left.{ }^{\star \star \star \star} p \leq 0.0001\right)$.

TABLE 3 | The 50\% cytotoxic concentration $\left(\mathrm{CC}_{50}\right)$ of free BNIPDaoct, BNIPDaoct-loaded emulsomes, free BNIPDanon, and BNIPDanon-loaded emulsomes on macrophages after 24,48 , and $72 \mathrm{~h}$ treatment.

\begin{tabular}{|c|c|c|c|c|}
\hline & \multicolumn{3}{|c|}{$\mathrm{CC}_{50}(\mu \mathrm{M})^{\mathrm{a}}$} & \multirow{2}{*}{$\frac{\mathrm{SI}\left(\mathrm{CC}_{50} / \mathrm{IC}_{50}\right)}{72 \mathrm{~h}}$} \\
\hline & $24 \mathrm{~h}$ & $48 h$ & $72 \mathrm{~h}$ & \\
\hline BNIPDaoct & $1.60 \pm 0.12$ & $1.49 \pm 0.03$ & $1.52 \pm 0.02$ & 1.81 \\
\hline BNIPDaoct-loaded emulsome & $15.85 \pm 0.06^{\star \star \star \star}$ & $18.16 \pm 0.19^{\star \star \star \star}$ & $17.73 \pm 0.03^{\star \star \star \star}$ & 30.1 \\
\hline BNIPDanon & $3.71 \pm 0.79$ & $3.26 \pm 1.15$ & $3.07 \pm 0.87$ & 1.7 \\
\hline BNIPDanon-loaded emulsome & $9.91 \pm 1.26^{\star \star \star \star}$ & $7.04 \pm 0.86^{\star \star \star \star}$ & $5.96 \pm 0.83^{\star \star \star}$ & 3.5 \\
\hline
\end{tabular}

a Viability determined by Alamar Blue assay. The results were obtained after treatment of the macrophages BNIPDaoct, BNIPDanon, and their emulsome formulations at concentrations (0.3-30 $\mu \mathrm{M})$ after 24, 48, and 72 h of incubation. Data represents mean $(\mathrm{n}=3) \pm S D$. Statistical significance was determined using two-way ANOVA, with Tukey's multiple-comparison test (ns: not significant; ${ }^{*} \mathrm{p} \leq 0.05,{ }^{* *} \mathrm{p} \leq 0.01,{ }^{* * *} \mathrm{p} \leq 0.001$, and ${ }^{* * * *} \mathrm{p} \leq 0.0001$ ).

recovery in macrophage viability was observed in between 48 and 72 -h cell viability data (i.e., from $6 \%$ to $13 \%$ and from $3 \%$ to $9 \%$ for 5 and $10 \mu \mathrm{M}$ BNIPDaoct concentrations, respectively). The incorporation of BNIPDanon into emulsomes decreased toxicity of the compound on healthy macrophages, as the data revealed that $\leq 3.75 \mu \mathrm{M}$ BNIPDanon-loaded emulsomes did not cause significant toxicity on healthy macrophages for $72 \mathrm{~h}$ (Figure 4D). However, when treated with $\geq 7.5 \mu \mathrm{M}$ BNIPDanon-loaded emulsomes the macrophage viability significantly decreased concentration-dependent manner below $25 \%, 14 \%$, and $8 \%$ after $72 \mathrm{~h}$, respectively (i.e., $7.5,15$, and $30 \mu \mathrm{M}$ concentrations) ( $p \leq 0.0001)$.

$\mathrm{CC}_{50}$ values were calculated for BNIPDaoct, BNIPDaoct-loaded emulsomes, BNIPDanon, and BNIPDanon-loaded emulsomes as $1.52 \pm 0.02 \mu \mathrm{M}, 17.73 \pm 0.03 \mu \mathrm{M}, 3.07 \pm$ $0.87 \mu \mathrm{M}$, and $5.96 \pm 0.83 \mu \mathrm{M}$, respectively (Table 3). These results indicated that the incorporation of BNIP derivatives into emulsomes significantly reduced their toxicities to 
A

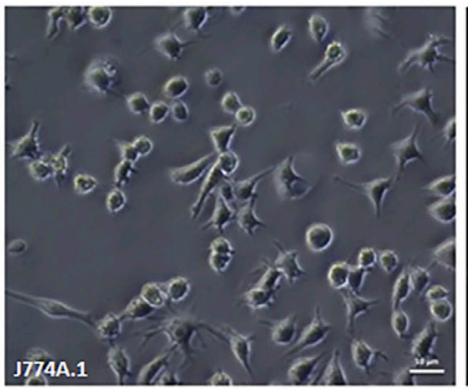

PKH26

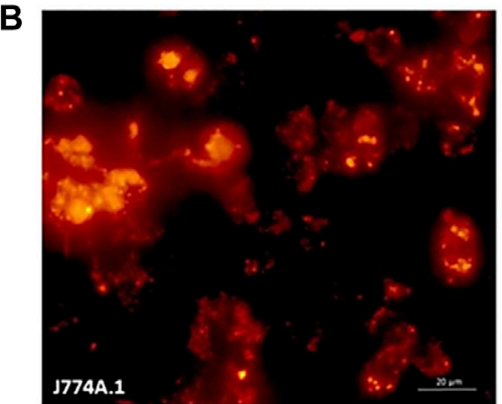

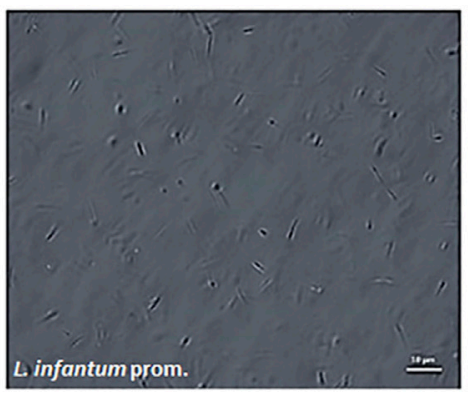

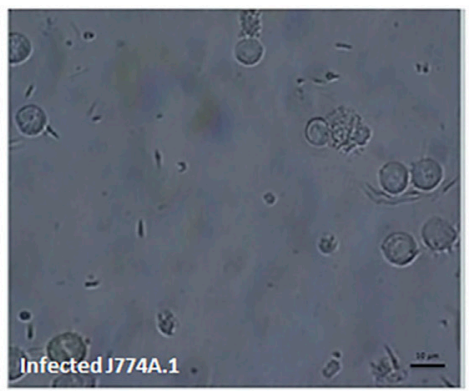

PKH67

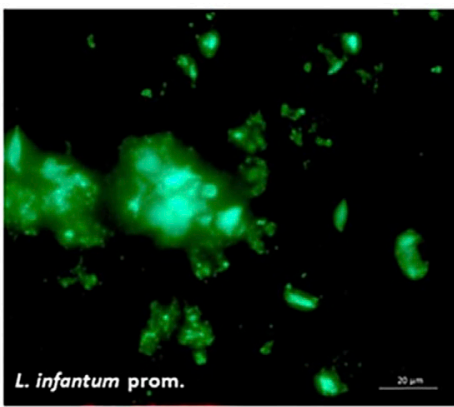

MERGED

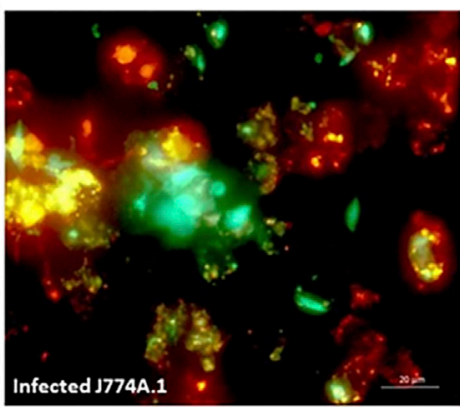

FIGURE 5 | Microscopy analysis of stained macrophage-promastigote co-culture (A) phase contrast and (B) fluorescence microscopy images of PKH26-stained macrophages (red) infected with PKH67-stained L. infantum promastigotes (green). The merged yellow color displays Leishmania-infected macrophages (magnification: $\times 63$; size bars: $20 \mu \mathrm{m})$
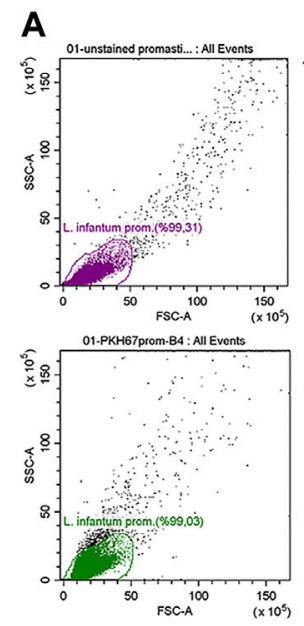
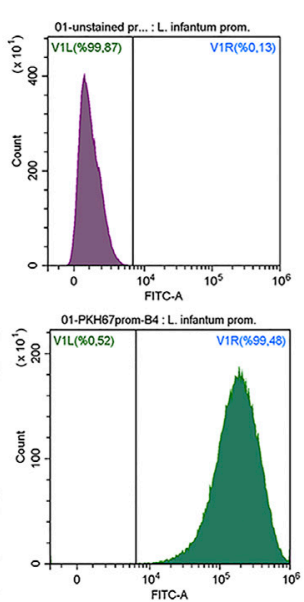

B

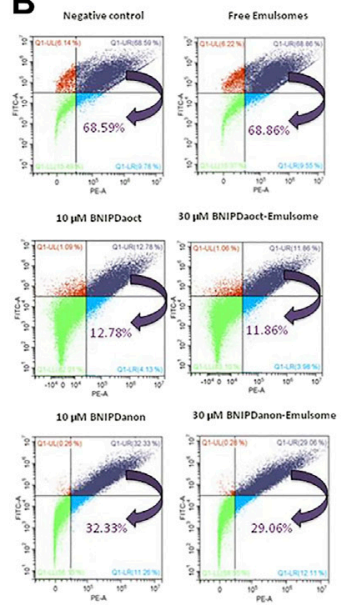

C
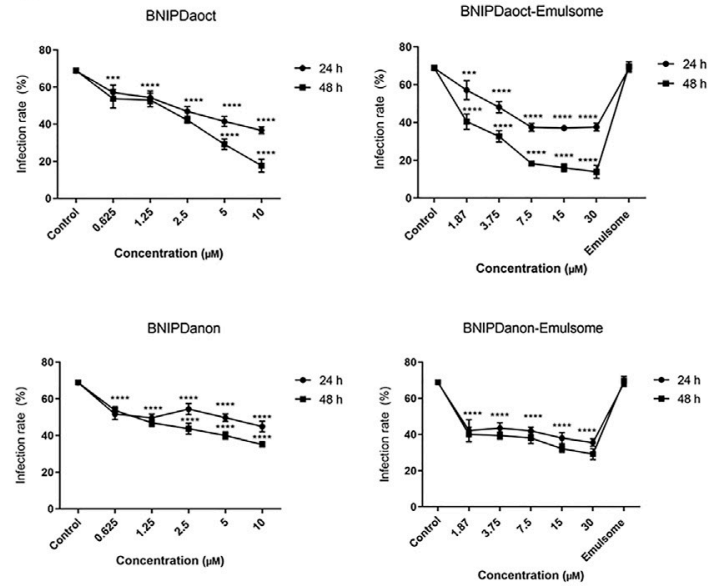

FIGURE 6 | (A) PKH67 staining. Percentage of unstained and staining achieved by PKH67-staining protocol on promastigotes as determined by flow cytometry. (B,C) Infection rates (\%) of macrophages with L. infantum promastigotes upon treatment with free BNIP and BNIP-loaded emulsomes as determined by flow cytometry (B) dot-plot data representation (for 48 h) and (C) graphical demonstration (24-48 h) for negative control, free BNIPDaoct, free BNIPDanon, BNIPDaoct-loaded, and BNIPDanon-loaded emulsome treatment groups. Data represents mean $(n=3) \pm$ SD. Statistical significance was determined using two-way ANOVA with Tukey's multiple-comparison test $\left({ }^{*} p \leq 0.05,{ }^{* *} p \leq 0.01,{ }^{\star \star *} p \leq 0.001\right.$, and $\left.{ }^{\star \star \star *} p \leq 0.0001\right)$.

macrophages, as demonstrated by higher $\mathrm{CC}_{50}$ values, i.e., $>11$-fold for BNIPDaoct $(p \leq 0.0001)$ and nearly 2 fold for BNIPDanon $(p \leq 0.001)$. In contrary to free BNIPDaoct, BNIPDaoct-loaded emulsomes showed no toxicity on macrophages at concentrations up to $15 \mu \mathrm{M}$ for 24, 48, and $72 \mathrm{~h}$. Moreover, BNIPDaoct-loaded emulsomes showed lower selectivity for macrophages, resulted in $>16$ fold increase in the selectivity index $\left(\mathrm{CC}_{50} / \mathrm{IC}_{50}\right)$ for $L$. 
infantum promastigotes ( $p \leq 0.0001)$ (Table 3). On the other hand, BNIPDanon-loaded emulsome indicated lower toxicity than its free form (Figure 4B), hence producing significant decrease (i.e., $\sim 2$-fold) in the selectivity of non-infected macrophages $(p \leq 0.001)$. It is important to report that free emulsomes did not show any significant toxicity on macrophages, when applied with concentrations equivalent to $30 \mu \mathrm{M}$ BNIP-loaded emulsomes (Figures 4B-D).

The obtained results revealed that emulsome formulations improved the efficacy of free forms of BNIPDaoct and BNIPDanon by reducing their cellular toxicity on macrophages. Among effective concentrations on $L$. infantum promastigotes, BNIPDaoct- and BNIPDanon-loaded emulsomes indicated the highest potential to inhibit parasite proliferation within the range of $0.5-15 \mu \mathrm{M}$ and $2-3.75 \mu \mathrm{M}$, respectively, without displaying any toxicity on macrophages (Figures 4B,D).

\subsubsection{Antileishmanial Effect of BNIP-loaded Emulsomes on Macrophages Infected by $L$. infantum Promastigotes}

Prior to the infection of macrophages with promastigotes, the parasites were stained with PKH67 to allow tracing by flow cytometry after the macrophage uptake.

Staining was applied on L. infantum promastigotes with PKH67 (green) and J774A.1 macrophages with PKH26 (red) dyes. Tracking the red, green, and yellow fluorescent signals in fluorescent microscopy images (Figure 5) and flow cytometry (Figure 6), it was possible to determine the populations of promastigotes, non-infected macrophages, and infected macrophages, respectively. Accordingly, Figure 5A displays the phase contrast images of J774A.1 macrophages infected by L. infantum promastigotes, where parasites inside and outside of the macrophages are visible. Figure 5B shows the fluorescent images of macrophages, where macrophages appear in red and promastigotes appear in green. The merged yellow color displays the macrophages infected by the parasites (Figure 5B).

Stained parasites were analyzed using flow cytometry analysis with a total of 100,000 events. Unstained parasites were determined as high as $99.87 \%$, where a negligible part of the population remained unstained (Figure 6A). As illustrated in Figure 6A, staining was successfully achieved for $99.48 \%$ of the promastigote population, leaving negligible number of promastigotes unstained. Similarly, macrophages were completely stained with $\mathrm{PKH} 26$ red fluorescence dyes (data not shown). These results suggest that the applied protocol and the concentration ratios for staining are effective for labeling the parasites and macrophages.

Flow cytometry analysis enabled the quantitative determination of the infection rates of macrophages. Antileishmanial activity of BNIPDaoct and BNIPDanon were studied on macrophage-promastigote co-culture for concentrations of $0.625,1.25,2.5,5$, and $10 \mu \mathrm{M}$ (Figures 6B,C). BNIPDaoct- and BNIPDanon-loaded emulsomes were tested on macrophage-promastigote co-culture for concentrations of 1.87, $3.75,7.5,15$, and $30 \mu \mathrm{M}$ BNIP derivatives. For instance, when macrophages were incubated with $L$. infantum promastigotes,
$68.6 \%$ of the macrophages become infected (Figure 6B). The treatment of Leishmania-infected macrophages with empty emulsomes (where the amount is equivalent to $30 \mu \mathrm{M}$ BNIPDaoct-loaded emulsome treatment group) for $48 \mathrm{~h}$ did not alter the infection rate (68.9\%). Treatment with $10 \mu \mathrm{M}$ BNIPDaoct and BNIPDanon for $48 \mathrm{~h}$ reduced the infection of macrophages to $12.8 \%$ and $32.3 \%$, respectively (Figure $6 \mathbf{B}$ ). Likewise, treatment with $30 \mu \mathrm{M}$ BNIPDaoct- and BNIPDanon-loaded emulsomes for $48 \mathrm{~h}$ significantly decreased the infection rates of macrophages to $11.9 \%(p \leq 0.0001)$ and $29.1 \%(p \leq 0.0001)$, respectively.

Twenty-fourhours of treatment with BNIPDaoct-loaded emulsome resulted in a significant reduction of the parasitic load at concentrations of $15 \mu \mathrm{M}(46.2 \%)$ and $7.5 \mu \mathrm{M}$ (45.6\%) compared to both free BNIPDaoct $(p \leq 0.001)$ and control groups $(p \leq 0.0001)$ (Figure 6C). Forty-eight hours of treatment with BNIPDaoct-loaded emulsome maintained reduction in the number of intracellular parasites at concentrations of $15 \mu \mathrm{M}$ (76.7\%) and $7.5 \mu \mathrm{M}$ (73.4\%) when compared to free BNIPDaoct and untreated macrophages. In contrary, treatment of infected macrophages with BNIPDanon-loaded emulsomes did not lead to a concentration- and time-dependent decrease in macrophage infection rate. Parasitic load decreased to $42.2 \%$ at $1.87 \mu \mathrm{M}$ BNIPDanon-loaded emulsome concentration at $24 \mathrm{~h}$ and remained almost stable regardless of further increase in both concentration and treatment time (Figure 6C).

Overall, the results obtained in the cell culture analysis demonstrated that the encapsulation of BNIPDaoct into emulsomes led to an increase in the efficacy of the compound to inhibit the growth of L. infantum parasites inside the macrophages. Morever, even at low doses, 7.5 $\mu \mathrm{M}$ BNIPDaoct-loaded emulsome and 1.87 $\mu \mathrm{M}$ BNIPDanon-loaded emulsome had a significant antileishmanial effect on Leishmania-infected macrophages ( $p \leq 0.0001$ ).

\section{DISCUSSION}

In the present study, BNIPDanon- and BNIPDaoct-loaded emulsomes were investigated for their ability to deliver the water-insoluble antileishmanial compounds to $L$. infantum parasites and the macrophages infected therewith. Emulsomes were selected as the drug delivery system in particular for their suitability for high loading capacity for hydrophobic substances such as BNIP compounds, high biocompatibility, controlled drug release profile, and accumulation preference in the organs of RES instead of the kidney, which largely reduces toxicity and is expected to contribute to the antileishmaniasis efficacy of the compounds. Characterization studies analyzed the physicochemical properties and the stability of the formulations, whereas the cell culture studies further explored the efficacy on both L. infantum and infected macrophages, as well as the safety of the formulation on non-infected macrophages.

SEM images displayed that BNIPDaoct- and BNIPDanonloaded emulsomes are spherical in shape with a smooth PL outermost surface (Figures 2B,C). The presence of BNIPDaoct and BNIPDanon-in the inner solid tripalmitin core of emulsomes and within the lipid bilayers surrounding the emulsomes-was demonstrated by confocal images, where 
incorporated BNIP derivatives with their autofluorescence property confirmed the encapsulation (Figure 2A).

The average diameters were determined as $363.1 \pm 51.7 \mathrm{~nm}$ and $337.4 \pm 50.8 \mathrm{~nm}$ for BNIPDaoct- and BNIPDanon-loaded emulsomes, respectively, where the plus-minus signs indicate the margin of average diameters. Comparing these values with the average particle size of empty emulsomes, i.e., $239.1 \pm 51.5 \mathrm{~nm}$, one can deduce that the encapsulation of BNIP derivatives within the emulsomes has significantly increased average particle size of the formulation (Table 1). This observation is parallel with the previous findings, suggesting that the incorporation of lipophilic compounds such as curcumin and piperine into emulsomes influences the average size of the lipid nanoparticles at a certain extent (Ucisik et al., 2013b; Ucisik et al., 2015b; Bolat et al., 2020). The alteration in particle size upon the incorporation of BNIP compounds may be attributed to the structural and physicochemical properties of BNIP compounds packed within the PLs and solid lipid core of the formulation. While the spatial arrangement of BNIP molecules inside the lipid composition may be considered as the main reason behind the increase in average size of emulsomes, BNIP compounds with two charged oxygen atoms may be generating additive electrostatic forces that also influence the spatial arrangement of the molecules in the composition (Pavlov et al., 2001). In contrast, Pal et al. observed in a previous study that the average size of AmpBloaded emulsomes increases, when the $\mathrm{AmpB}$ concentration in the formulation is decreased ( $\mathrm{Pal}$ et al., 2012). This controversial behavior was attributed to the association of AmpB molecules with cholesterol inside the PL bilayers (Vyas et al., 2000; Pal et al., 2012). Likewise, the interaction of BNIP compounds with cholesterol or DPPC may have contributed to the alteration in size of emulsomes upon encapsulation, but, in this instance, leading to an increase in average size.

Average zeta potential of BNIPDaoct- and BNIPDanonloaded emulsomes $(-4.77 \pm 6.68 \mathrm{mV}$ and $-10.1 \pm 9.76 \mathrm{mV}$, respectively) were observed to be less negative compared to empty emulsomes $(-16.74 \pm 4.56 \mathrm{mV}$ ) (Table 1). Despite confirming the shift in zeta potential upon encapsulation toward a neutral charge, the statistical analysis indicated that the difference between the average values is not significant $(p \geq$ 0.05 ). In our opinion, a zeta potential value close to neutral might be considered as a reason or the outcome of increased particle size after encapsulation. For instance, the inverse correlation between the average size and the average zeta potential of nanoparticles was observed and reported previously (du Plessis et al., 1994).

The particle size requirements for drug delivery systems vary according to the route of administration (Shekunov et al., 2007). Among the various administration routes, transdermal drug delivery can be suitable for emulsomes having a particle size within the range of 150-300 $\mathrm{nm}$. Previous studies suggested that 50- and 500-nm nanoparticles that are negatively charged allow a high charge density of contact with the skin because the particle sizes between 50 and $500 \mathrm{~nm}$ are able to enhance permeation of the epidermis through the stratum corneum (Kohli and Alpar, 2004). du Plessis et al. (1994) demonstrated that the intermediate particle size of $300 \mathrm{~nm}$ resulted in both the highest reservoir in the deeper skin layers and the highest drug concentration in the reservoir, confirming that the topical drug delivery is influenced by the size of liposomes (du Plessis et al., 1994; Verma et al., 2003). Beside transdermal route, in the intravenous administration, nanocarriers in the range of $150-200 \mathrm{~nm}$ can be accumulated easily by the RES, especially by the Kupffer cells in the liver, which largely reduces systemic toxicity of the antileishmanial therapy and improves the efficacy of the antileishmanial compounds (Chaubey et al., 2014). Size larger than $300 \mathrm{~nm}$ should be avoided due to problems such as thrombosis and rapid elimination through the liver and spleen. Accordingly, an optimum delivery system with a size ranging from 30 to $300 \mathrm{~nm}$ should be precisely adjusted regarding to the route of administration under consideration (Ferrari et al., 2018). In light of this information, BNIP-loaded emulsomes with average sizes above $300 \mathrm{~nm}$ were regarded to be suitable for the in vitro cell culture study to evaluate the efficacy of the prepared formulation on $L$. infantum promastigotes and macrophages infected therewith. It is also important to emphasize that the size of emulsomes can be further tuned by the PL-to-TL ratio, as it was previously reported that each decrease in the PL:TL ratio between 0.14 and 0.4 yields formation of emulsomes with smaller sizes (Heiati et al., 1996; Vyas et al., 2006; Ucisik et al., 2013a; Ucisik et al., 2013b; Ucisik et al., 2015a).

In the study, emulsomes were selected as the nanocarrier system for their high loading capacity for lipophilic compounds (Ucisik et al., 2015a; Amselem et al., 2018). The $\mathrm{IC}_{50}$ values of the compounds were previously reported as $0.78 \pm$ $0.05 \mu \mathrm{M}$ for BNIPDaoct and $0.78 \pm 0.10 \mu \mathrm{M}$ for BNIPDanon against $L$. infantum promastigotes (Oliveira et al., 2007). In the current study, the encapsulation of the compounds within emulsomes yielded highest $(0.31 \mathrm{mg} / \mathrm{ml} ; 0.5 \mathrm{mM})$ BNIPDaoct and $(0.24 \mathrm{mg} / \mathrm{ml} ; 0.38 \mathrm{mM})$ BNIPDanon concentrations, where both values are considered as sufficient to achieve effective concentrations in both $L$. infantum parasites and infected macrophages.

The incorporation of BNIPDaoct and BNIPDanon into emulsomes improved the efficacy of the compound against $L$. infantum promastigotes. For instance, while $0.5 \mu \mathrm{M}$ BNIPDaoct achieved a decrease in the viability of $L$. infantum promastigotes to approximately $50 \%$, when provided within emulsome, the cell viability became approximately $20.0 \%$. Likewise, $2 \mu \mathrm{M}$ BNIPDanon decreased the viability of $L$. infantum promastigotes to approximately $30 \%$, whereas BNIPDanonloaded emulsome at the same concentration reduced the viability to approximately $10 \%$ (Figures 3A,B). Empty emulsomes did not show any effect on proliferation of $L$. infantum promastigotes for $72 \mathrm{~h}$, indicating that the observed antileismanial activity of the formulations directly stems from the BNIP compounds carried by the emulsomes.

The potency of the BNIP-loaded emulsomes in inhibiting the proliferation of $L$. infantum promastigote was investigated via the estimated $\mathrm{IC}_{50}$ values. Accordingly, the incorporation of the BNIPDaoct on emulsomes led to a significant improvement ( $p \leq$ $0.01)$ on the $\mathrm{IC}_{50}$ value $(0.84 \pm 0.09 \mu \mathrm{M})$ for the promastigotes compared to the free compound $(0.59 \pm 0.08 \mu \mathrm{M})$, whereas no significant change was shown between the $\mathrm{IC}_{50}$ value of BNIPDanon $(1.85 \pm 0.01 \mu \mathrm{M})$ and BNIPDanon-loaded 
emulsomes $(1.73 \pm 0.02 \mu \mathrm{M})$ (Table 2). In the literature, the $\mathrm{IC}_{50}$ value for BNIPDaoct molecule-as tested on $L$. infantum promastigotes-was given as $0.78 \pm 0.05 \mu \mathrm{M}$ (Oliveira et al., 2007), which was found to be comparable with the value realized in our study, i.e., $0.84 \pm 0.09 \mu \mathrm{M}$ (Table 2). In contrast, the $\mathrm{IC}_{50}$ value for BNIPDanon $(1.85 \pm 0.01 \mu \mathrm{M})$ was found different than the value in the literature, i.e., $0.78 \pm 0.10 \mu \mathrm{M}$ (Oliveira et al., 2007). The higher $\mathrm{IC}_{50}$ value of the BNIPDanon compound synthesized and studied in our study might be attributed to the differences in the synthesis methodology of the compound. For instance, in their study, Oliveira et al. have adjusted the salt ratio (i.e., di- or trihydrobromide salts) in the buffer to enhance the aqueous solubility of active ingredients (Oliveira et al., 2007), whereas the synthesis of both BNIPDaoct and BNIPDanon in our study did not require the addition of salt for this purpose. In a previous study on naphthalimides and bis-naphthalimidesare classes of compounds including BNIPs demonstrated that the antileishmanial activity is dependent on a second naphthilimide group in the molecule (Silva et al., 2016). The optimal distance between the two naphthalimidopropylamine groups that results in the most potent inhibitory activity toward LiSIR2RP1 was observed with the linker group, which contains nine methylene units, i.e., BNIDanon (Tavares et al., 2010).

Cell viability studies on macrophages revealed that emulsomes do not expose any toxicity to the macrophages. When delivered within the emulsomes, both BNIP compounds displayed reduced toxicities (Figure 4). The delivery of the compounds within the emulsomes increased the selectivity of BNIPDaoct and BNIPDanon for L. infantum promastigotes as the ratio of $\mathrm{CC}_{50} / \mathrm{IC}_{50}$ by $>16$ - and $\sim 2$-fold, respectively. The most striking result to emerge from the data was the approximately 30.1 -fold increase in selectivity of BNIPDaoctloaded emulsomes for $L$. infantum against macrophages (Table 3).

These results have further strengthened the hypothesis that emulsomes in the lack of any surfactants but the presence of their lipid composition have the potential to contribute to the safety of the BNIP compounds on healthy macrophages. Moreover, it is foremost important to highlight that free emulsomes did not show any significant toxicity on macrophages even at concentrations equivalent to $30 \mu \mathrm{M}$ BNIPDaoct-loaded emulsome treatment, distinguishing emulsomes once again as a safe and biocompatible drug delivery system.

Infection of macrophages by $L$. infantum promastigotes were evaluated through both fluorescence microscopy and dualfluorescent flow cytometry analysis as described previously (Islek et al., 2021). The use of flow cytometry for accurate quantification of the infection rates on macrophages was previously reported (Di Giorgio et al., 2000; Islek et al., 2021). This applied methodology brought the advantage of rapid analysis of high cell numbers compared to the alternative microscopic approaches. Likewise, PKH67 green and PKH26 red fluorescence dyes were used in our study to label $L$. infantum promastigotes and J774 A.1 macrophages, respectively, and infection rates of macrophages were then estimated as percentage of number of promastigotes per macrophages. High success rate in selective staining of the promastigotes and macrophages (99.48\%) verified the reliability of the applied methodology (Figure 6).

Flow cytometry analysis verified that emulsomes do not interfere with the Leishmania infection of the macrophages. In contrast, BNIPDaoct-loaded emulsomes led to a significant reduction in the parasitic load at concentrations above $7.5 \mu \mathrm{M}$ compared to the negative control group ( $p \leq 0.0001$ ) (Figure 6C). However, BNIPDanon-loaded emulsomes did not cause any evident decrease in macrophage infection rates in concentration- and time-dependent manner. Instead, at $1.87 \mu \mathrm{M}$ BNIPDanon-loaded emulsome concentration for $24 \mathrm{~h}$, the infection rate first decreased to nearly $40 \%$ and did not alter significantly despite the increase in both concentration level and treatment duration (Figure 6C). These results suggest that the incorporation of BNIPDaoct and BNIPDanon into emulsomes enhanced the efficacy and selectivity of the compounds for L. infantum intracellular parasites inside the macrophages. The data provided have further strengthened our hypothesis that emulsomes with their lipid characteristics and safety feature have the potential to contribute to BNIP compounds in their inherited toxicity against macrophages.

As this study presented, emulsomes have the ability to overcome the solubility limitation of the compounds and to improve the safety on macrophages. Among the two emulsome formulations, BNIPDaoct-loaded emulsomes showed better activity against both extracellular and intracellular $L$. infantum parasites and increased selectivity for L. infantum promastigotes compared to macrophages. Composed of a solid tripalmitin core and PL outermost surface, emulsomes were successful in providing high efficacy in treatment.

\section{CONCLUSION}

BNIPDaoct and BNIPDanon are bis-naphthalimidesare molecules that recently came forward with antileishmanial activities beyond the standard, commercialized AmpB therapy (Costa Lima et al., 2012b; Tavares et al., 2012). However, the exposed high-level toxicity on macrophages and the hydrophobic characteristics, leading to low water solubility and bioavailability, necessitate the use of drug delivery systems, where otherwise medical use remains limited. The presented study introduced BNIPDaoct- and BNIPDanon-loaded emulsomes as promising lipid-based drug delivery systems to address these limitations.

In summary, the incorporation of BNIP compounds into emulsomes results in a stable drug delivery system that achieves the delivery of BNIPDaoct and BNIPDanon into $L$. infantum parasites and macrophages. The promising antileishmanial efficacy of BNIP-loaded emulsomes on in vitro model highlights the potential of the antileishmanial therapy in future in vivo and clinical studies. It is important to emphasize that this study is the first report revealing emulsomes as the nanocarrier system capable to enhance the antileishmanial therapy with BNIPDaoct and BNIPDanon on both L. infantum parasites and Leishmania-infected macrophages. Moreover, the safety feature of BNIP-loaded emulsomes can be further enhanced by surface modifications for active targeting to further improve the antileishmanial activity and the selectivity of the formulation. 


\section{DATA AVAILABILITY STATEMENT}

The original contributions presented in the study are included in the article/supplementary material. Further inquiries can be directed to the corresponding author.

\section{AUTHOR CONTRIBUTIONS}

MG, FS, and MU together constructed the study, provided funding for the research, and guided the study. Synthesis of BNIP derivatives was achieved by EK and BS under the supervision of MG. ZI and MU designed the framework of the material characterization and cell culture studies. ZI produced BNIP-loaded emulsomes, carried out the characterization studies, and performed the entire cell culture studies. MU and ZI analyzed and interpreted the data of the cell culture studies. AG-A and AT contributed to the design and implementation of the research on parasite culture and further contributed to the editing of the manuscript. FS provided additional resources to complete cell culture experiments. FS, MU and ZI were actively involved in writing and revising the manuscript. All authors have read and approved the final manuscript.

\section{REFERENCES}

Adler-moore, J. P., and Proffitt, R. T. (2008). Amphotericin B Lipid Preparations: What Are the Differences? Clin. Microbiol. Infect. 14, 25-36. doi:10.1111/ j.1469-0691.2008.01979.x

Al-Natour, S. H. (2009). Update in the Treatment of Cutaneous Leishmaniasis. J. Fam. Community Med. 16, 41-47. Available at: https://www.ncbi.nlm.nih. gov/pmc/articles/PMC3377028/.

Alhakamy, N. A., Badr-Eldin, S. M., Ahmed, O. A. A., Asfour, H. Z., Aldawsari, H. M., Algandaby, M. M., et al. (2020). Piceatannol-loaded Emulsomes Exhibit Enhanced Cytostatic and Apoptotic Activities in colon Cancer Cells. Antioxidants 9, 419. doi:10.3390/antiox9050419

Amselem, S., Zawoznik, E., Yogev, A., and Friedman, D. (2018). "Emulsomes, A New Type of Lipid Assembly," in Handbook of Nonmedical Applications of Liposomes: Volume III: From Design to Microreactors (Boca Raton, FL: CRC Press), 209-224. doi:10.1201/9781351072724-12

Aronson, N. E. (2017). Addressing a Clinical challenge: Guidelines for the Diagnosis and Treatment of Leishmaniasis. BMC Med. 15, 76. doi:10.1186/ s12916-017-0843-3

Bolat, Z. B., Islek, Z., Demir, B. N., Yilmaz, E. N., Sahin, F., and Ucisik, M. H. (2020). Curcumin- and Piperine-Loaded Emulsomes as Combinational Treatment Approach Enhance the Anticancer Activity of Curcumin on HCT116 Colorectal Cancer Model. Front. Bioeng. Biotechnol. 8, 50. doi:10.3389/fbioe. 2020.00050

Chakravarty, J., and Sundar, S. (2010). Drug Resistance in Leishmaniasis. J. Glob. Infect Dis 2, 167. doi:10.4103/0974-777x.62887

Chaubey, P., Patel, R. R., and Mishra, B. (2014). Development and Optimization of Curcumin-Loaded Mannosylated Chitosan Nanoparticles Using Response Surface Methodology in the Treatment of Visceral Leishmaniasis. Expert Opin. Drug Deliv. 11, 1163-1181. doi:10.1517/17425247.2014.917076

Costa Lima, S. A., Resende, M., Silvestre, R., Tavares, J., Ouaissi, A., Lin, P. K. T., et al. (2012a). Characterization and Evaluation of BNIPDaoct-Loaded PLGA Nanoparticles for Visceral Leishmaniasis: In Vitro and In Vivo Studies. Nanomedicine 7, 1839-1849. doi:10.2217/nnm.12.74

Costa Lima, S., Rodrigues, V., Garrido, J., Borges, F., Kong Thoo Lin, P., and Cordeiro Da Silva, A. (2012b). In Vitro evaluation of Bisnaphthalimidopropyl Derivatives Loaded into Pegylated Nanoparticles against Leishmania Infantum Protozoa. Int. J. Antimicrob. Agents 39, 424-430. doi:10.1016/j.ijantimicag.2012.01.003

\section{FUNDING}

Integrated to the COST action CM1307 "Targeted chemotherapy towards diseases caused by endoparasites", this study was financially supported by the Scientific and Technological Research Council of Turkey (TUBITAK) under the 2515 EU-COST grant with project title "Discovery of Novel Drug Molecules and Development of Nanoparticular Drug Delivery Systems for Treatment of Leishmaniasis" and number 115Z846. Yeditepe University Department of Genetics and Bioengineering provided additional support to cell culture studies.

\section{ACKNOWLEDGMENTS}

The authors kindly appreciate TUBITAK (115Z846) and EU-COST organization (COST action CM1307). The authors would like to thank Dr. Esra Albayrak for her assistance at flow cytometry. The authors also thank Sadik Bay for his assistance at imaging of emulsomes in SEM.

Croft, S. L., and Olliaro, P. (2011). Leishmaniasis Chemotherapy-Challenges and Opportunities. Clin. Microbiol. Infect. 17, 1478-1483. doi:10.1111/j.14690691.2011.03630.x

Croft, S. L., Sundar, S., and Fairlamb, A. H. (2006). Drug Resistance in Leishmaniasis. Clin. Microbiol. Rev. 19, 111-126. doi:10.1128/CMR.19.1.111126.2006

Date, A., Joshi, M., and Patravale, V. (2007). Parasitic Diseases: Liposomes and Polymeric Nanoparticles versus Lipid Nanoparticles 2 . Adv. Drug Deliv. Rev. 59, 505-521. doi:10.1016/j.addr.2007.04.009

Di Giorgio, C., Ridoux, O., Delmas, F., Azas, N., Gasquet, M., and Timon-David, P. (2000). Flow Cytometric Detection of Leishmania Parasites in Human Monocyte-Derived Macrophages: Application to Antileishmanial-Drug Testing. Antimicrob. Agents Chemother. 44, 3074-3078. doi:10.1128/ AAC.44.11.3074-3078.2000

du Plessis, J., Ramachandran, C., Weiner, N., and Müller, D. G. (1994). The Influence of Particle Size of Liposomes on the Deposition of Drug into Skin. Int. J. Pharm. 103, 277-282. doi:10.1016/0378-5173(94)90178-3

El-Zaafarany, G., Soliman, M., Mansour, S., Cespi, M., Palmieri, G., Illum, L., et al. (2018). A Tailored Thermosensitive PLGA-PEG-PLGA/emulsomes Composite for Enhanced Oxcarbazepine Brain Delivery via the Nasal Route. Pharmaceutics 10, 217. doi:10.3390/pharmaceutics10040217

Ferrari, R., Sponchioni, M., Morbidelli, M., and Moscatelli, D. (2018). Polymer Nanoparticles for the Intravenous Delivery of Anticancer Drugs: The Checkpoints on the Road from the Synthesis to Clinical Translation. Nanoscale 10, 22701-22719. doi:10.1039/c8nr05933k

Freitas-Junior, L. H., Chatelain, E., Kim, H. A., and Siqueira-Neto, J. L. (2012) Visceral Leishmaniasis Treatment: What Do We Have, what Do We Need and How to Deliver it? Int. J. Parasitol. Drugs Drug Resist. 2, 11-19. doi:10.1016/ j.ijpddr.2012.01.003

Gill, V., Kumar, M. S., Khurana, B., Arora, D., and Mahadevan, N. (2011). Development of Amphotericin B Loaded Modified Emulsomes for Visceral Leishmaniasis. Vitr. Int. J. Recent Adv. Pharm. Res. 1, 14-20.

Gomes-Alves, A. G., Maia, A. F., Cruz, T., Castro, H., and Tomás, A. M. (2018). Development of an Automated Image Analysis Protocol for Quantification of Intracellular Forms of Leishmania Spp. PLoS One 13, e0201747. doi:10.1371/ journal.pone.0201747

Gupta, S., Dube, A., and Vyas, S. P. (2012). Development and Characterization of Amphotericin B Loaded Solid Lipid Nanoparticles against Experimental Visceral Leishmaniasis. Pnt 1, 54-67. doi:10.2174/ 2211738511301010054 
Gupta, S., Dube, A., and Vyas, S. P. (2007). Antileishmanial Efficacy of Amphotericin B Bearing Emulsomes against Experimental Visceral Leishmaniasis. J. Drug Target. 15, 437-444. doi:10.1080/10611860701453836

Gupta, S., and Vyas, S. P. (2007). Development and Characterization of Amphotericin B Bearing Emulsomes for Passive and Active Macrophage Targeting. J. Drug Target. 15, 206-217. doi:10.1080/10611860701195395

Heiati, H., Phillips, N. C., and Tawashi, R. (1996). Evidence for Phospholipid Bilayer Formation in Solid Lipid Nanoparticles Formulated with Phospholipid and Triglyceride. Pharm. Res. 13, 1406-1410. doi:10.1023/A: 1016090420759

Heiati, H., Tawashi, R., and Phillips, N. C. (1998). Drug Retention and Stability of Solid Lipid Nanoparticles Containing Azidothymidine Palmitate after Autoclaving, Storage and Lyophilization. J. Microencapsulation 15, 173-184. doi:10.3109/02652049809006847

Heiati, H., Tawashi, R., Shivers, R. R., and Phillips, N. C. (1997). Solid Lipid Nanoparticles as Drug Carriers I. Incorporation and Retention of the Lipophilic Prodrug 3'-Azido-3'-Deoxythymidine Palmitate. Int. J. Pharm. 146, 123-131. doi:10.1016/S0378-5173(96)04782-5

Islek, Z., Ucisik, M. H., and Sahin, F. (2021). Novel Dual-Fluorescent Flow Cytometric Approach for Quantification of Macrophages Infected with Leishmania Infantum Parasites. Parasitology, 1-7. doi:10.1017/ S0031182021001530

Jain, K., and Jain, N. K. (2013). Novel Therapeutic Strategies for Treatment of Visceral Leishmaniasis. Drug Discov. Today 18, 1272-1281. doi:10.1016/ j.drudis.2013.08.005

Köse, Ș., Töz, S. Ö., Lu, İ. C. B. Ğ., Olut, A., Korkmaz, M., and Özbel, Y. (2004). Case Report : Treatment of Kala-Azar by Amphotericin B Lipid Complex (ABELCET ${ }^{\circledR}$ ). 28, 126-128.

Kammath, A. J., Nair, B., P, S., and Nath, L. R. (2020). Curry versus Cancer: Potential of Some Selected Culinary Spices against Cancer with In Vitro, In Vivo, and Human Trials Evidences. J. Food Biochem. 45, 1-21. doi:10.1111/ jfbc. 13285

Keskin, E., Ucisik, M. H., Sucu, B. O., and Guzel, M. (2019). Novel Synthetic Approaches for Bisnaphthalimidopropyl (BNIP) Derivatives as Potential Antiparasitic Agents for the Treatment of Leishmaniasis. Molecules 24, 4607. doi:10.3390/molecules24244607

Kohli, A. K., and Alpar, H. O. (2004). Potential Use of Nanoparticles for Transcutaneous Vaccine Delivery: Effect of Particle Size and Charge. Int. J. Pharmaceutics 275, 13-17. doi:10.1016/j.ijpharm.2003.10.038

Kretschmar, M., Amselem, S., Zawoznik, E., Mosbach, K., Dietz, A., Hof, H., et al. (2001). Efficient Treatment of Murine Systemic Infection with Candida Albicans Using Amphotericin B Incorporated in Nanosize Range Particles (Emulsomes). Mycoses, 281-286. doi:10.1046/j.1439-0507.2001.00654.x

Kumari, S., Kumar, A., Samant, M., Singh, N., and Dube, A. (2008). Discovery of Novel Vaccine Candidates and Drug Targets against Visceral Leishmaniasis Using Proteomics and Transcriptomics. Cdt 9, 938-947. doi:10.2174/ 138945008786786091

Lowell, G. H., Kaminski, R. W., VanCott, T. C., Slike, B., Kersey, K., Zawoznik, E., et al. (1997). Proteosomes, Emulsomes, and Cholera Toxin B Improve Nasal Immunogenicity of Human Immunodeficiency Virus Gp160 in Mice: Induction of Serum, Intestinal, Vaginal, and Lung IgA and IgG. J. Infect. Dis. 175, 292-301. doi:10.1093/infdis/175.2.292

Makala, L. H. C., and Baban, B. (2014). "Novel Therapeutic Approaches to Leishmania Infection," in Leishmaniasis - Trends in Epidemiology, Diagnosis and Treatment. Rijeka, Croatia: IntechOpen. doi:10.5772/58167

Mohamed-Ahmed, A. H. A., Brocchini, S., and Croft, S. L. (2012). Recent Advances in Development of Amphotericin B Formulations for the Treatment of Visceral Leishmaniasis. Curr. Opin. Infect. Dis. 25, 695-702. doi:10.1097/ QCO.0b013e328359eff2

Murray, H. W., Berman, J. D., Davies, C. R., and Saravia, N. G. (2005). Advances in Leishmaniasis. Lancet 366 (9496), 1561-1577. doi:10.1016/S0140-6736(05) 67629-5

Natera, S., Machuca, C., Padrón-Nieves, M., Romero, A., Díaz, E., and Ponte-Sucre, A. (2007). Leishmania spp.: Proficiency of Drug-Resistant Parasites. Int. J. Antimicrob. Agents 29, 637-642. doi:10.1016/j.ijantimicag.2007.01.004

Oliveira, J., Ralton, L., Tavares, J., Codeiro-da-Silva, A., Bestwick, C. S., McPherson, A., et al. (2007). The Synthesis and the In Vitro Cytotoxicity Studies of Bisnaphthalimidopropyl Polyamine Derivatives against colon Cancer Cells and Parasite Leishmania Infantum. Bioorg. Med. Chem. 15, 541-545. doi:10.1016/j.bmc.2006.09.031

Oliveira, M., Gomes-Alves, A. G., Sousa, C., Mirta Marani, M., Plácido, A., Vale, N., et al. (2016). Ocellatin-PT Antimicrobial Peptides: High-Resolution Microscopy Studies in Antileishmania Models and Interactions with Mimetic Membrane Systems. Biopolymers 105, 873-886. doi:10.1002/bip.22925

Ouellette, M., Drummelsmith, J., and Papadopoulou, B. (2004). Leishmaniasis: Drugs in the Clinic, Resistance and New Developments. Drug Resist. Updates 7, 257-266. doi:10.1016/j.drup.2004.07.002

Pal, A., Gupta, S., Jaiswal, A., Dube, A., and Vyas, S. P. (2012). Development and Evaluation of Tripalmitin Emulsomes for the Treatment of Experimental Visceral Leishmaniasis. J. Liposome Res. 22, 62-71. doi:10.3109/08982104.2011.592495

Paliwal, R., Paliwal, S. R., Mishra, N., Mehta, A., and Vyas, S. P. (2009). Engineered Chylomicron Mimicking Carrier Emulsome for Lymph Targeted Oral Delivery of Methotrexate. Int. J. Pharmaceutics 380, 181-188. doi:10.1016/j.ijpharm.2009.06.026

Pavlov, V., Kong Thoo Lin, P., and Rodilla, V. (2001). Cytotoxicity, DNA Binding and Localisation of Novel Bis-Naphthalimidopropyl Polyamine Derivatives. Chem. Biol. Interact. 137, 15-24. doi:10.1016/S0009-2797(01)00165-X

Rodrigues, V., Cordeiro-Da-Silva, A., Laforge, M., Silvestre, R., and Estaquier, J. (2016). Regulation of Immunity during Visceral Leishmania Infection. Parasites Vectors 9, 118. doi:10.1186/s13071-016-1412-x

Shekunov, B. Y., Chattopadhyay, P., Tong, H. H. Y., and Chow, A. H. L. (2007). Particle Size Analysis in Pharmaceutics: Principles, Methods and Applications. Pharm. Res. 24, 203-227. doi:10.1007/s11095-006-9146-7

Silva, A., Alves, M., Thoo-Lin, P., Baptista, C., Noro, J., Cristo, G., et al. (2016). Exploiting the Antiparasitic Activity of Naphthalimides Derivatives. Int J. Chem. Pharm. Sci. 4, 19-23.

Smith, A. C., Yardley, V., Rhodes, J., and Croft, S. L. (2000). Activity of the Novel Immunomodulatory Compound Tucaresol against Experimental Visceral Leishmaniasis. Antimicrob. Agents Chemother. 44, 1494-1498. doi:10.1128/ AAC.44.6.1494-1498.2000

Sundar, S., and Chakravarty, J. (2013). Leishmaniasis: An Update of Current Pharmacotherapy. Expert Opin. Pharmacother. 14, 53-63. doi:10.1517/ 14656566.2013.755515

Sundar, S., and Chatterjee, M. (2006). Visceral Leishmaniasis - Current Therapeutic Modalities. Indian J. Med. Res. 123, 345-352.

Sundar, S., and Jaya, J. (2010). Liposomal Amphotericin B and Leishmaniasis: Dose and Response. J. Glob. Infect Dis 2, 159. doi:10.4103/0974-777x.62886

Sundar, S., Jha, T. K., Thakur, C. P., Engel, J., Sindermann, H., Fischer, C., et al. (2002a). Oral Miltefosine for Indian Visceral Leishmaniasis. N. Engl. J. Med. 347, 1739-1746. doi:10.1056/nejmoa021556

Sundar, S., Pai, K., Sahu, M., Kumar, V., and Murray, H. W. (2002b). Immunochromatographic Strip-Test Detection of Anti-K39 Antibody in Indian Visceral Leishmaniasis. Ann. Trop. Med. Parasitol. 96, 19-23. doi:10.1179/000349802125000466

Sundar, S., Jha, T. K., Thakur, C. P., Mishra, M., Singh, V. P., and Buffels, R. (2003). Single-Dose Liposomal Amphotericin B in the Treatment of Visceral Leishmaniasis in India: A Multicenter Study. Clin. Infect. Dis. 37, 800-804. doi:10.1086/377542

Sundar, S., Mehta, H., Suresh, A. V., Singh, S. P., Rai, M., and Murray, H. W. (2004). Amphotericin B Treatment for Indian Visceral Leishmaniasis: Conventional versus Lipid Formulations. Clin. Infect. Dis. 38, 377-383. doi:10.1086/380971

Tavares, J., Ouaissi, A., Lin, P. K. T., Loureiro, I., Kaur, S., Roy, N., Cordeiro-daSilva, A., et al. (2010). Bisnaphthalimidopropyl Derivatives as Inhibitors of Leishmania SIR2 Related Proteinâ€ 1. ChemMedChem 5, 140-147. doi:10.1002/ cmdc. 200900367

Tavares, J., Ouaissi, A., Lin, P. K. T., Tomás, A., and Cordeiro-Da-Silva, A. (2005). Differential Effects of Polyamine Derivative Compounds against Leishmania Infantum Promastigotes and Axenic Amastigotes. Int. J. Parasitol. 35, 637-646. doi:10.1016/j.ijpara.2005.01.008

Tavares, J., Ouaissi, A., Silva, A. M., Lin, P. K. T., Roy, N., and Cordeiro-da-Silva, A. (2012). Anti-leishmanial Activity of the Bisnaphthalimidopropyl Derivatives. Parasitol. Int. 61, 360-363. doi:10.1016/.jparint.2011.11.005

Tiuman, T. S., Santos, A. O., Ueda-Nakamura, T., Filho, B. P. D., and Nakamura, C. V. (2011). Recent Advances in Leishmaniasis Treatment. Int. J. Infect. Dis. 15, e525-e532. doi:10.1016/j.ijid.2011.03.021

Ucisik, M. H., Küpcü, S., Debreczeny, M., Schuster, B., and Sleytr, U. B. (2013a). S-layer Coated Emulsomes as Potential Nanocarriers. Small 9, 2895-2904. doi:10.1002/smll.201203116 
Ucisik, M. H., Küpcü, S., Schuster, B., and Sleytr, U. B. (2013b). Characterization of CurcuEmulsomes: Nanoformulation for Enhanced Solubility and Delivery of Curcumin. J. Nanobiotechnology 11, 37. doi:10.1186/1477-3155-11-37

Ucisik, M., Sleytr, U., and Schuster, B. (2015a). Emulsomes Meet S-Layer Proteins: An Emerging Targeted Drug Delivery System. Curr. Pharm. Biotechnol. 16, 392-405. doi:10.2174/138920101604150218112656

Ucisik, M. H., Küpcü, S., Breitwieser, A., Gelbmann, N., Schuster, B., and Sleytr, U. B. (2015b). S-layer Fusion Protein as a Tool Functionalizing Emulsomes and CurcuEmulsomes for Antibody Binding and Targeting. Colloids Surf. B: Biointerfaces 128, 132-139. doi:10.1016/j.colsurfb.2015.01.055

Verma, D. D., Verma, S., Blume, G., and Fahr, A. (2003). Particle Size of Liposomes Influences Dermal Delivery of Substances into Skin. Int. J. Pharm. 258, 141-151. doi:10.1016/S0378-5173(03)00183-2

Vyas, S. P., Katare, Y. K., Mishra, V., and Sihorkar, V. (2000). Ligand Directed Macrophage Targeting of Amphotericin B Loaded Liposomes. Int. J. Pharm. 210, 1-14. doi:10.1016/S0378-5173(00)00522-6

Vyas, S. P., Khatri, K., and Goyal, A. K. (2009). Functionalized Nanocarrier(s) to Image and Target Fungi Infected Immune Cells. Med. Mycol. 47, S362-S368. doi:10.1080/13693780802464430

Vyas, S. P., Subhedar, R., and Jain, S. (2010). Development and Characterization of Emulsomes for Sustained and Targeted Delivery of an Antiviral Agent to Liver. J. Pharm. Pharmacol. 58, 321-326. doi:10.1211/jpp.58.3.0005
World Health Organization (2013). Sustaining the Drive to Overcome the Global Impact of Neglected Tropical Diseases. World Heal. Organ.

Yilmaz, E. N., Bay, S., Ozturk, G., and Ucisik, M. H. (2020). Neuroprotective Effects of Curcumin-Loaded Emulsomes in a Laser Axotomy-Induced CNS Injury Model. Ijn Vol. 15, 9211-9229. doi:10.2147/IJN.S272931

Conflict of Interest: The authors declare that the research was conducted in the absence of any commercial or financial relationships that could be construed as a potential conflict of interest.

Publisher's Note: All claims expressed in this article are solely those of the authors and do not necessarily represent those of their affiliated organizations or those of the publisher, the editors, and the reviewers. Any product that may be evaluated in this article, or claim that may be made by its manufacturer, is not guaranteed or endorsed by the publisher.

Copyright (C) 2022 Islek, Ucisik, Keskin, Sucu, Gomes-Alves, Tomás, Guzel and Sahin. This is an open-access article distributed under the terms of the Creative Commons Attribution License (CC BY). The use, distribution or reproduction in other forums is permitted, provided the original author(s) and the copyright owner(s) are credited and that the original publication in this journal is cited, in accordance with accepted academic practice. No use, distribution or reproduction is permitted which does not comply with these terms. 